hep-th/0103144

\title{
Free Field Construction for the Eight-Vertex Model: Representation for Form Factors
}

\author{
Michael Lashkevich \\ Landau Institute for Theoretical Physics, \\ 142432 Chernogolovka, Russia
}

\begin{abstract}
The free field realization of the eight-vertex model is extended to form factors. It is achieved by constructing offdiagonal with respect to the ground state sectors matrix elements of the $\Lambda$ operator which establishes a relation between corner transfer matrices of the eight-vertex model and of the SOS model. As an example, the two-particle form factor of the $\sigma^{z}$ operator is evaluated explicitly.
\end{abstract}

March 2001

\section{Introduction}

This paper continues the research started in [1,2], where an approach to calculating local correlation functions of the eight-vertex/XYZ model based on the vertex operator approach (see [3] and references therein) and the vertex-face correspondence [4] was proposed. Here the method is extended to provide a representation for form factors. As an example, explicit expressions for the two-particle form factor of the $\sigma^{z}$ operator have been found.

Form factors are matrix elements of local operators in the basis of eigenvectors of the transfer matrix or Hamiltonian. In principle, all physical properties of a system can be expressed in terms of form factors. In practice, form factors provide series for correlation functions well converging off the critical point and finite expressions for some other quantities of physical importance.

In the case of the six-vertex model the integral representation for correlation functions and form factors is based on the infinite-dimensional symmetry of the model on the infinite lattice, described by the quantum affine algebra $U_{q}\left(\widehat{s l}_{2}\right)$, and on the free field representation for the elements of the algebra and the intertwining operators of its infinite-dimensional representations. The free boson field takes its origin in the Cartan-type elements of the algebra in the Drinfeld realization. In the case of the eight-vertex model the Drinfeld realization of its symmetry algebra, $\mathcal{A}_{q, p}\left(\widehat{s l}_{2}\right)[5]$, is unknown.

It was shown [1] that the correlation functions of the eight-vertex model can be related to those of the solid-on-solid (SOS) model with an insertion of a particular non-local operator $\Lambda$. As the free field representation for the SOS model was known [6] it remained to find the free field realization of the $\Lambda$ operator. Such a realization was found for the particular matrix elements diagonal in the SOS ground state sector. Though that was enough for correlation functions, the operators related to excitations in the eightvertex model being mapped onto the SOS model contain non-zero elements off-diagonal in ground state sector. So the construction for form factors remained unsolved problem. It is solved here.

The structure of the paper is the following. In Sec. 2 the definitions of the models are reminded and main notations are introduced. In Sec. 3 the vertex operator algebra that unifies the eight-vertex and SOS models in the corner transfer matrix representation is described. In Sec. 4 the free field realization of the $\Lambda$ operator is proposed which is the main result of the paper. The simplest form factor is computed in Sec. 5. Details of some calculations and some formulas used in the paper are gathered in Appendices. 


$$
R(u-v)_{\varepsilon_{1} \varepsilon_{2}}^{\varepsilon_{3} \varepsilon_{4}}=\left.v \stackrel{\varepsilon_{2}}{\left.\right|_{u} ^{\varepsilon_{1}}}\right|_{u} ^{\varepsilon_{3}} \varepsilon_{4}
$$

(a)

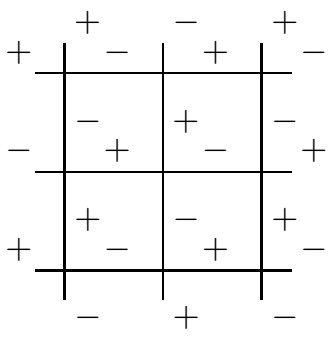

(b)

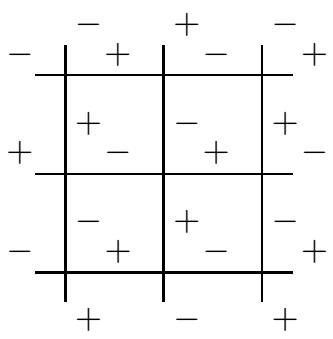

Fig. 1. Eight-vertex model: (a) definition of the weight matrix; (b) two degenerate ground states.

\section{Basic models and vertex-face correspondence}

Recall that the eight-vertex model [7] is a model of classical statistical mechanics on a square lattice, where the fluctuating variables ('spins') $\varepsilon= \pm \equiv \pm 1$ are associated to links of the lattice, while the interaction is associated to vertices. Namely, a Boltzmann weight $R_{\varepsilon_{1} \varepsilon_{2}}^{\varepsilon_{3} \varepsilon_{4}}$ is associated to each vertex of the model (Fig. 1a). The total statistical weight of the configuration of spin variables is proportional to the product of local Boltzmann weights over the lattice. As a Boltzmann weight $R_{\varepsilon_{1} \varepsilon_{2}}^{\varepsilon_{3} \varepsilon_{4}}$ is assumed to be nonzero for $\varepsilon_{1}+\varepsilon_{2}+\varepsilon_{3}+\varepsilon_{4}=0 \quad(\bmod 4)$ only, there are eight admissible configurations around each vertex, which justifies the name of the model. If, besides, the weights are invariant under simultaneous change of all signs of spins, the model is integrable [9] and this is the case under consideration. The nonzero Boltzmann weights are

$$
a=R_{++}^{++}=R_{--}^{--}, \quad b=R_{+-}^{+-}=R_{-+}^{-+}, \quad c=R_{+-}^{-+}=R_{-+}^{+-}, \quad d=R_{++}^{--}=R_{--}^{++} .
$$

The $R$ matrix can be parametrized in elliptic functions. Namely, consider it as a function $R(u) \equiv R(u ; \epsilon, r)$ of the spectral parameter $u$ and two additional parameters $\epsilon$ and $r$ with matrix elements

$$
\begin{aligned}
a(u ; \epsilon, r) & =\rho(u ; \epsilon, r) s(1-u ; \epsilon, r), \\
b(u ; \epsilon, r) & =\rho(u ; \epsilon, r) s(u ; \epsilon, r), \\
c(u ; \epsilon, r) & =\rho(u ; \epsilon, r) s(1 ; \epsilon, r), \\
d(u ; \epsilon, r) & =\rho(u ; \epsilon, r) s(1-u ; \epsilon, r) s(u ; \epsilon, r) s(1 ; \epsilon, r),
\end{aligned}
$$

where

$$
s(u ; \epsilon, r)=\frac{\theta_{1}\left(\frac{u}{2 r} ; \frac{\mathrm{i} \pi}{2 \epsilon r}\right)}{\theta_{2}\left(\frac{u}{2 r} ; \frac{\mathrm{i} \pi}{2 \epsilon r}\right)} .
$$

Here $\theta_{i}(u ; \tau)(i=1, \ldots, 4)$ are the Jacobi theta functions with the quasiperiods 1 and $\tau(\operatorname{Im} \tau>0)$.

We shall also use the multiplicative variables

$$
x=\mathrm{e}^{-\epsilon}, \quad p=x^{2 r}, \quad z=x^{2 u}
$$

on equal grounds with the additive ones $\epsilon, r, u$.

The common factor $\rho(u ; \epsilon, r)$ is convenient to be fixed so that the partition function per site were equal to one. Under this condition it reads

$$
\begin{gathered}
\rho(u ; \epsilon, r)=x^{1-r / 2} \frac{\left(x^{2 r+2} ; x^{4 r}\right)_{\infty}\left(x^{2 r-2} ; x^{4 r}\right)_{\infty}}{\left(x^{2 r} ; x^{4 r}\right)_{\infty}^{2}} \frac{\left(x^{2 r} z ; x^{4 r}\right)_{\infty}\left(x^{2 r} z^{-1} ; x^{4 r}\right)_{\infty}}{\left(x^{4 r-2} z ; x^{4 r}\right)_{\infty}\left(x^{2} z^{-1} ; x^{4 r}\right)_{\infty}} \frac{g\left(z^{-1}\right)}{g(z)} \\
g(z) \equiv g(z ; \epsilon, r)=\frac{\left(x^{2} z ; x^{4}, x^{2 r}\right)_{\infty}\left(x^{2 r+2} z ; x^{4}, x^{2 r}\right)_{\infty}}{\left(x^{4} z ; x^{4}, x^{2 r}\right)_{\infty}\left(x^{2 r} z ; x^{4}, x^{2 r}\right)_{\infty}} \\
\left(z ; p_{1}, \ldots, p_{N}\right)_{\infty}=\prod_{n_{1}, \ldots, n_{N}=0}^{\infty}\left(1-z p_{1}^{n_{1}} \ldots p_{N}^{n_{N}}\right) .
\end{gathered}
$$

The matrix $R(u)$ satisfies the Yang-Baxter equation

$$
R_{12}\left(u_{1}-u_{2}\right) R_{13}\left(u_{1}-u_{3}\right) R_{23}\left(u_{2}-u_{3}\right)=R_{23}\left(u_{2}-u_{3}\right) R_{13}\left(u_{1}-u_{3}\right) R_{12}\left(u_{1}-u_{2}\right),
$$




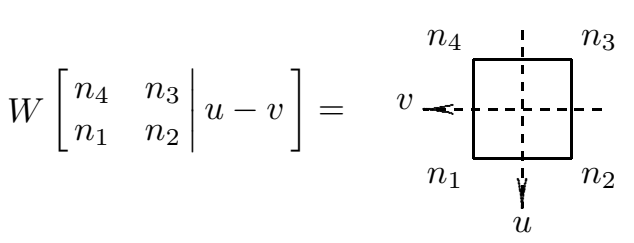

(a)

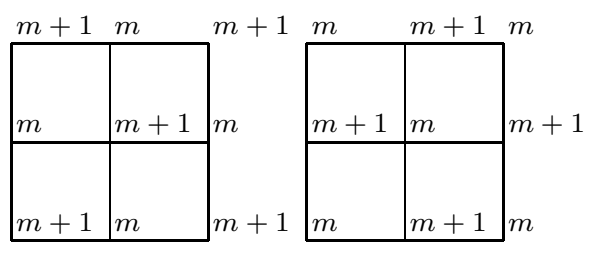

(b)

Fig. 2. SOS model: (a) definition of weights; $(b)$ an infinite number of degenerate ground states labeled by $m \in \mathbf{Z}+\delta$ subject to $(k-1) r<m, m+1<k r$ for some integer $k$ and by position of the lesser value.

where $R_{i j}(u): V_{i} \otimes V_{j} \rightarrow V_{i} \otimes V_{j}$ acting on a tensor product of several copies of identical spaces $V_{i} \simeq \mathbf{C}^{2}$, and, with the common factor chosen in the form (2.3), the unitarity and crossing relations:

$$
R_{12}(u) R_{21}(-u)=\mathrm{id}, \quad R(u)_{\varepsilon_{1} \varepsilon_{2}}^{\varepsilon_{3} \varepsilon_{4}}=R(1-u)_{\varepsilon_{4},-\varepsilon_{1}}^{\varepsilon_{2},-\varepsilon_{3}}
$$

The region

$$
\epsilon>0, \quad r>1, \quad-1<u<1
$$

corresponds to the 'antiferroelectric' region $a+|b|+|d|<c$. There are two ground state configurations shown in Fig. $1 \mathrm{~b}$ in this region. In these ground states the $c$-type vertex configurations are only realized. Any other configuration with finite energy differs from one of these ground states by flopping a finite number of spins. We shall speak about two ground state sectors in this model.

We shall consider the inhomogeneous generalization of the eight-vertex model (the $Z$-invariant eightvertex model [8]) in which a value of the spectral parameter is associated to each line of the model and the difference of spectral parameters at a vertex enters the respective $R$ matrix (Fig. 1a).

Let us formulate now the solid-on-solid (SOS) model. This model was proposed by Baxter [4] as a partition function preserving twist of the eight-vertex model that admits solution by means of the Bethe Ansatz.

In the SOS model a fluctuating variable ('height') is associated to each site of the lattice and runs over the set $\mathbf{Z}+\delta$ with some $\delta$ satisfying $0<\delta<1$. The partition function is $\delta$ independent. We shall consider $\delta$ as a fixed parameter. The heights $n_{1}$ and $n_{2}$ at adjacent sites satisfy the admissibility condition

$$
\left|n_{1}-n_{2}\right|=1 \text {. }
$$

Interaction is described by the Boltzmann weights $W\left[\begin{array}{ll}n_{4} & n_{3} \\ n_{1} & n_{2}\end{array} \mid u\right]$ associated to the faces of the lattice, while the spectral parameters are attached to the lines of the dual lattice (Fig. 2a):

$$
\begin{aligned}
& W\left[\begin{array}{cc}
n & n \pm 1 \\
n \pm 1 & n \pm 2
\end{array} \mid u\right]=R_{0}(u), \\
& W\left[\begin{array}{cc|c}
n & n \pm 1 \\
n \pm 1 & n & u
\end{array}\right]=R_{0}(u) \frac{[n \pm u][1]}{[n][1-u]}, \\
& W\left[\begin{array}{cc|}
n & n \pm 1 \\
n \mp 1 & n
\end{array} \mid u\right]=-R_{0}(u) \frac{[n \pm 1][u]}{[n][1-u]} .
\end{aligned}
$$

Here

$$
R_{0}(u) \equiv R_{0}(u ; \epsilon, r)=z^{(r-1) / 2 r} \frac{g\left(z^{-1}\right)}{g(z)}
$$

with $g(z)$ from (2.3) and here and below we use the assignments

$$
\begin{aligned}
{[u]_{i} } & =h_{i}(u ; r)=\sqrt{\frac{\pi}{\epsilon r}} \mathrm{e}^{\frac{1}{4} \epsilon r} \theta_{i}\left(\frac{u}{r} ; \frac{\mathrm{i} \pi}{\epsilon r}\right), \\
{[u] } & =[u]_{1}=x^{u^{2} / r-u}(z ; p)_{\infty}\left(p z^{-1} ; p\right)_{\infty}(p ; p)_{\infty} .
\end{aligned}
$$

For brevity, we omitted the variables $\epsilon$ and $r$ in the argument of the $W \mathrm{~s}$, but we shall restore them when necessary. Generally, for any function $f$ of these variables we shall imply $f(u) \equiv f(u ; \epsilon, r)$. 


$$
\begin{aligned}
& t_{\varepsilon}\left(u_{0}-u\right)_{n}^{n^{\prime}}=\frac{n^{\prime}}{u_{0} \propto---}
\end{aligned}
$$

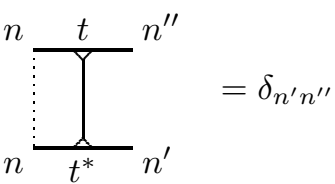

$$
\begin{aligned}
& t_{\varepsilon}^{*}\left(u_{0}-u\right)_{n}^{n^{\prime}}=u_{0} \frac{\mid}{n} \frac{\varepsilon}{y_{u}^{\dot{1}}} n^{\prime} \\
& n^{\prime \prime} \frac{\prod^{\prime}}{\prod^{*}}{ }_{n}^{n}=\delta_{n^{\prime} n^{\prime \prime}}
\end{aligned}
$$

Fig. 3. Intertwining vectors: $(a)$ graphic notations; $(b)$ definition of the conjugate and 'primed' vectors.

The weights $W(u)$ satisfy the dynamical Yang-Baxter equation and, with the normalization (2.6), the dynamical versions of the unitarity and crossing conditions. The advantage of the SOS model is absence of a $d$-type configuration, which makes it possible to apply the techniques developed for the six-vertex/XXZ model.

We are interested in the so called regime III:

$$
\epsilon>0, \quad r \geq 1, \quad 0<u<1 \text {. }
$$

There is an infinite number of degenerate, but inequivalent, ground states in this regime shown in Fig. $2 \mathrm{~b}$. The ground state sectors will be labelled by the value of $m$. The two pictures of Fig. $2 \mathrm{~b}$ are distinguished by parity of $m-n$ with $n$ being a value of the height at some given site.

Consider the functions (Fig. 3a)

$$
\begin{aligned}
& t_{+}(u)_{n}^{n^{\prime}}=\mathrm{i}^{1 / 2} f(u) \theta_{3}\left(\frac{\left(n^{\prime}-n\right) u+n^{\prime}}{2 r} ; \mathrm{i} \frac{\pi}{2 \epsilon r}\right), \\
& t_{-}(u)_{n}^{n^{\prime}}=\mathrm{i}^{-1 / 2}(-1)^{n-m_{0}+1} f(u) \theta_{4}\left(\frac{\left(n^{\prime}-n\right) u+n^{\prime}}{2 r} ; \mathrm{i} \frac{\pi}{2 \epsilon r}\right)
\end{aligned}
$$

with $m_{0}$ being an arbitrary number from $\mathbf{Z}+\delta$ and $n, n^{\prime}$ being an admissible pair of height variables. It is convenient to choose the common factor $f(u)$ so that

$$
[u] f(u) f(u-1)=C \equiv \frac{[0]_{4}^{2}}{2 \theta_{3}(0 ; \mathrm{i} \pi / 2 \epsilon r) \theta_{4}(0 ; \mathrm{i} \pi / 2 \epsilon r)} .
$$

In [1] $f(u)$ was chosen in the form

$$
\begin{aligned}
f(u) & =\sqrt{C} x^{-u^{2} / 2 r+(r-1) / 2 r+1 / 4} f_{1}\left(x^{2 u}\right) \\
f_{1}(z) & =\frac{1}{\sqrt{\left(x^{2 r} ; x^{2 r}\right)_{\infty}}} \frac{\left(x^{4} z ; x^{4}, x^{2 r}\right)_{\infty}\left(x^{2+2 r} z^{-1} ; x^{4}, x^{2 r}\right)_{\infty}}{\left(x^{2} z ; x^{4}, x^{2 r}\right)_{\infty}\left(x^{2 r} z^{-1} ; x^{4}, x^{2 r}\right)_{\infty}}
\end{aligned}
$$

The functions $t_{\varepsilon}(u)_{n}^{n^{\prime}}$ (called intertwining vectors) relate the vertex and face type $R$ matrices (Fig. 4a):

$$
\sum_{\varepsilon_{1}^{\prime} \varepsilon_{2}^{\prime}} R(u-v)_{\varepsilon_{1} \varepsilon_{2}}^{\varepsilon_{2}^{\prime} \varepsilon_{2}^{\prime}} t_{\varepsilon_{1}^{\prime}}\left(u_{0}-u\right)_{s^{\prime}}^{n^{\prime}} t_{\varepsilon_{2}^{\prime}}\left(u_{0}-v\right)_{n}^{s^{\prime}}=\sum_{s \in \mathbf{Z}} t_{\varepsilon_{2}}\left(u_{0}-v\right)_{s}^{n^{\prime}} t_{\varepsilon_{1}}\left(u_{0}-u\right)_{n}^{s} W\left[\begin{array}{cc}
n^{\prime} & s^{\prime} \\
s & n
\end{array} \mid u-v\right]
$$

The last relation is referred to as the vertex-face correspondence.

It is convenient to introduce the 'conjugate' and 'primed' intertwining vectors (Fig. 3b):

$$
\sum_{\varepsilon} t_{\varepsilon}^{*}(u)_{n}^{n^{\prime}} t_{\varepsilon}(u)_{n^{\prime \prime}}^{n}=\delta_{n^{\prime} n^{\prime \prime}}, \quad \sum_{\varepsilon} t_{\varepsilon}^{*}(u)_{n^{\prime}}^{n} t_{\varepsilon}^{\prime}(u)_{n}^{n^{\prime \prime}}=\delta_{n^{\prime} n^{\prime \prime}}
$$




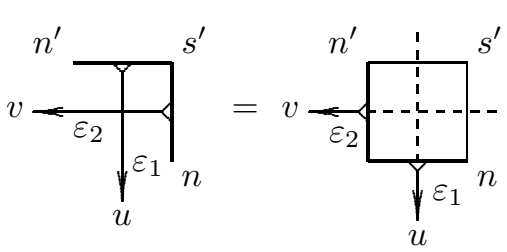

(a)

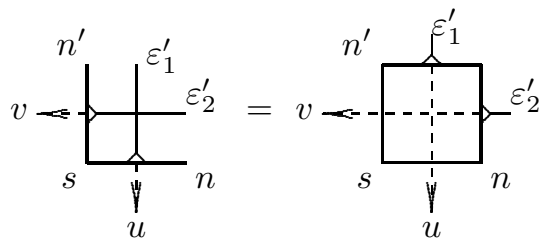

(b)

Fig. 4. Vertex-face correspondence: (a) by usual intertwining vectors; (b) by conjugate intertwining vectors.

Explicitly, with $f(u)$ subjected to $(2.10)$ we have

$$
\begin{aligned}
t_{\varepsilon}^{*}(u)_{n}^{n^{\prime}} & =(-)^{n-m_{0}+1} \frac{\left(n^{\prime}-n\right)}{[n]} t_{-\varepsilon}(u-1)_{n}^{n^{\prime}}, \\
t_{\varepsilon}^{\prime}(u)_{n}^{n^{\prime}} & =\frac{\left[n^{\prime}\right]}{[n]} t_{\varepsilon}(u-2)_{n}^{n^{\prime}} .
\end{aligned}
$$

The conjugate intertwining vector enters another form of the vertex-face correspondence (Fig. 4b):

$$
\sum_{\varepsilon_{1} \varepsilon_{2}} t_{\varepsilon_{2}}^{*}\left(u_{0}-v\right)_{n^{\prime}}^{s} t_{\varepsilon_{1}}^{*}\left(u_{0}-u\right)_{s}^{n} R(u-v)_{\varepsilon_{1} \varepsilon_{2}}^{\varepsilon_{1}^{\prime} \varepsilon_{2}^{\prime}}=\sum_{s^{\prime} \in \mathbf{Z}} W\left[\begin{array}{cc}
n^{\prime} & s^{\prime} \\
s & n
\end{array} \mid u-v\right] t_{\varepsilon_{1}^{\prime}}^{*}\left(u_{0}-u\right)_{n^{\prime}}^{s^{\prime}} t_{\varepsilon_{2}^{\prime}}^{*}\left(u_{0}-v\right)_{s^{\prime}}^{n} .
$$

For $t_{\varepsilon}^{\prime}(u)_{n}^{n^{\prime}}$ the identity of the form $(2.12)$ is valid.

\section{Vertex operator algebra}

Algebras of vertex operators describe models of statistical mechanics in the corner transfer matrix approach (see [3] and references therein). Let us recall the structures of these algebras for the eight-vertex and SOS models $[10,11]$.

For a given orthogonal basis $|+\rangle$ and $|-\rangle$ in $\mathbf{C}^{2}$, consider the subspace $\mathcal{H}_{i}$ in $\mathbf{C}^{2} \otimes \mathbf{C}^{2} \otimes \cdots$ spanned on the vectors $\left|\varepsilon_{1}\right\rangle \otimes\left|\varepsilon_{2}\right\rangle \otimes \cdots$ such that $\varepsilon_{k}$ stabilizes to $(-)^{k+i}$ for large $k$. It means that the number $i=0,1$ labels the ground state sector. We shall identify this space with a right half row of vertical links on the lattice. All operators are thought to act in the upper direction on the right half lattice.

Define the operators

$$
\rho^{(i)}=x^{4 H^{(i)}}: \mathcal{H}_{i} \rightarrow \mathcal{H}_{i}, \quad \Phi_{\varepsilon}^{(1-i, i)}(u), \Psi_{\varepsilon}^{*(1-i, i)}(u): \mathcal{H}_{i} \rightarrow \mathcal{H}_{1-i} .
$$

The spectrum of the corner Hamiltonian $H^{(i)}$ is equidistant and is determined by the generating function

$$
\chi(q) \equiv \operatorname{Tr}_{\mathcal{H}_{0}} q^{H^{(0)}}=\operatorname{Tr}_{\mathcal{H}_{1}} q^{H^{(1)}}=\left(q^{1 / 2} ; q\right)_{\infty}^{-1} .
$$

In the $H^{(i)}$-graded basis the matrix elements of the operators $\Phi$ and $\Psi^{*}$ are meromorphic functions of the parameters $z^{1 / 2}=x^{u}$ and $z=x^{2 u}$, respectively, with the only pole at $z^{1 / 2}=0(z=0)$.

The vertex operators $\Phi_{\varepsilon}^{(1-i, i)}(u), \Psi_{\varepsilon}^{*(1-i, i)}(u)$ satisfy the commutation relations ${ }^{a}$

$$
\begin{gathered}
\Phi_{\varepsilon_{1}}^{(i, 1-i)}\left(u_{1}\right) \Phi_{\varepsilon_{2}}^{(1-i, i)}\left(u_{2}\right)=\sum_{\varepsilon_{1}^{\prime} \varepsilon_{2}^{\prime}} R\left(u_{1}-u_{2}\right)_{\varepsilon_{1} \varepsilon_{2}}^{\varepsilon_{1}^{\prime} \varepsilon_{2}^{\prime}} \Phi_{\varepsilon_{2}^{\prime}}^{(i, 1-i)}\left(u_{2}\right) \Phi_{\varepsilon_{1}^{\prime}}^{(1-i, i)}\left(u_{1}\right), \\
\sum_{\varepsilon_{1}^{\prime} \varepsilon_{2}^{\prime}} \tilde{R}\left(u_{1}-u_{2}\right)_{\varepsilon_{1} \varepsilon_{2}}^{\varepsilon_{1}^{\prime} \varepsilon_{2}^{\prime}} \Psi_{\varepsilon_{1}^{\prime}}^{*(i, 1-i)}\left(u_{1}\right) \Psi_{\varepsilon_{2}^{\prime}}^{*(1-i, i)}\left(u_{2}\right)=\Psi_{\varepsilon_{2}}^{*(i, 1-i)}\left(u_{2}\right) \Psi_{\varepsilon_{1}}^{*(1-i, i)}\left(u_{1}\right), \\
\Phi_{\varepsilon_{1}}^{(i, 1-i)}\left(u_{1}\right) \Psi_{\varepsilon_{2}}^{*(1-i, i)}\left(u_{2}\right)=\tau\left(u_{1}-u_{2}\right) \Psi_{\varepsilon_{2}}^{*(i, 1-i)}\left(u_{2}\right) \Phi_{\varepsilon_{1}}^{(1-i, i)}\left(u_{1}\right)
\end{gathered}
$$

\footnotetext{
${ }^{a} \mathrm{Up}$ to now this is not a theorem, but a well-checked conjecture [12].
} 
and the operator $\rho^{(i)}$ shifts the spectral parameter:

$$
\Phi_{\varepsilon}^{(1-i, i)}(u) \rho^{(i)}=\rho^{(1-i)} \Phi_{\varepsilon}^{(1-i, i)}(u-2), \quad \Psi_{\varepsilon}^{*(1-i, i)}(u) \rho^{(i)}=\rho^{(1-i)} \Psi_{\varepsilon}^{*(1-i, i)}(u-2) .
$$

Here $\tilde{R}(u)$ is the $R$ matrix with the matrix elements

$$
\tilde{a}(u)=-a(u ; \epsilon, r-1), \quad \tilde{b}(u)=-b(u ; \epsilon, r-1), \quad \tilde{c}(u)=-c(u ; \epsilon, r-1), \quad \tilde{d}(u)=d(u ; \epsilon, r-1),
$$

and

$$
\tau(u)=\mathrm{i} \frac{\theta_{1}\left(\frac{1}{4}-\frac{u}{2} ; \frac{\mathrm{i} \pi}{2 \epsilon}\right)}{\theta_{1}\left(\frac{1}{4}+\frac{u}{2} ; \frac{\mathrm{i} \pi}{2 \epsilon}\right)} .
$$

Besides, the vertex operators satisfy the additional relations

$$
\begin{gathered}
\sum_{\varepsilon} \Phi_{\varepsilon}^{*(i, 1-i)}(u) \Phi_{\varepsilon}^{(1-i, i)}(u)=1, \quad \Phi_{\varepsilon_{1}}^{(i, 1-i)}(u) \Phi_{\varepsilon_{2}}^{*(1-i, i)}(u)=\delta_{\varepsilon_{1} \varepsilon_{2}}, \\
\Psi_{\varepsilon_{1}}^{(i, 1-i)}\left(u^{\prime}\right) \Psi_{\varepsilon_{2}}^{*(1-i, i)}(u)=\frac{1}{\pi} \frac{\delta_{\varepsilon_{1} \varepsilon_{2}}}{u^{\prime}-u}+O\left(\left(u^{\prime}-u\right)^{0}\right),
\end{gathered}
$$

where

$$
\Phi_{\varepsilon}^{*(1-i, i)}(u)=\Phi_{-\varepsilon}^{(1-i, i)}(u-1), \quad \Psi_{\varepsilon}^{(1-i, i)}(u)=\Psi_{-\varepsilon}^{*(1-i, i)}(u-1) .
$$

Below we will usually omit the superscripts indicating the ground state sector and write simply $\rho, \Phi_{\varepsilon}(u)$ etc.

The operator $\rho$ is nothing but the product of four corner transfer matrices of the model. The operators $\Phi_{\varepsilon}(u)$ (type I vertex operators) form the 'half transfer matrix' which is a half-infinite version of the monodromy operator. Products of the operators $\Psi_{\varepsilon}(u)$ (type II vertex operators) represent many-particle eigenstates of the transfer matrix. The function $\tau(u)$ describes the excitation spectrum of the model while the function $\tilde{R}(u)$ is the two-particle $S$ matrix of the elementary excitations.

Let $\left|a_{1} \theta_{1}, \ldots, a_{N} \theta_{N}\right\rangle^{(i)}$ be an eigenvector of the transfer matrix corresponding to $N$ 'charged' particles with charges $a_{j}$ and 'rapidities' $\theta_{j}=\pi^{2} / 2 \epsilon+\mathrm{i} \pi v_{j}$, normalized by the condition

$$
{ }^{(i)}\left\langle a_{1}^{\prime} \theta_{1}^{\prime}, \ldots, a_{N}^{\prime} \theta_{N}^{\prime} \mid a_{1} \theta_{1}, \ldots, a_{N} \theta_{N}\right\rangle^{(i)}=(2 \pi)^{N} \sum_{\sigma \in S_{N}} \prod_{j=1}^{N} \delta_{a_{\sigma(j)}^{\prime} a_{j}} \delta\left(\theta_{\sigma(j)}^{\prime}-\theta_{j}\right) .
$$

'Rapidities' are implied to be real numbers and defined modulo $\pi^{2} / \epsilon$. The vacuum state, which corresponds to $N=0$, will be designated as $|0\rangle^{(i)}$. For $r<2$ this basis is incomplete because of presence of 'neutral' breather-like bound states. Nevertheless, the form factors with these bound states are expressed in terms of the form factors that only contain 'charged' particles, though with complex rapidities.

Let $\mathcal{H}_{i, i}$ be a subspace of the tensor product $\cdots \otimes \mathbf{C}^{2} \otimes \mathbf{C}^{2} \otimes \cdots$ spanned on the vectors $\cdots \otimes\left|\varepsilon_{1}\right\rangle \otimes\left|\varepsilon_{2}\right\rangle \otimes \cdots$ such that $\varepsilon_{k}=(-)^{k+i+1}$ for $k \rightarrow \infty$ and for $k \rightarrow-\infty$. We shall identify this space with a column of horizontal links in the lattice of the eight-vertex model. Let $E_{\varepsilon^{\prime} \varepsilon}: \mathbf{C}^{2} \rightarrow \mathbf{C}^{2}$ be a matrix with the only nonzero matrix element $\left(\varepsilon^{\prime}, \varepsilon\right)$ equal to 1 . Respectively, the operator $E_{\varepsilon^{\prime} \varepsilon}^{(n)}: \mathcal{H}_{i, i} \rightarrow \mathcal{H}_{i, i}$ acts as $E_{\varepsilon^{\prime} \varepsilon}$ on the $n$th tensor component of $\mathcal{H}_{i, i}$ and as the identity mapping on other components. To the operator

$$
\mathcal{O}=E_{\varepsilon_{1}^{\prime} \varepsilon_{1}}^{(1)} \ldots E_{\varepsilon_{M}^{\prime} \varepsilon_{M}}^{(M)}
$$

in $\mathcal{H}_{i, i}$ we assign the operator

$$
\hat{\mathcal{O}}=\Phi_{\varepsilon_{M}^{\prime}}^{*}\left(u_{M}\right) \ldots \Phi_{\varepsilon_{1}^{\prime}}^{*}\left(u_{1}\right) \Phi_{\varepsilon_{1}}\left(u_{1}\right) \ldots \Phi_{\varepsilon_{M}}\left(u_{M}\right)
$$

in $\mathcal{H}_{i}$. The meaning of the arguments $u_{j}$ is the following. We attach the same fixed value of the spectral parameter to each vertical line and the zero spectral parameter to each horizontal line except these $M$ lines where the values $-u_{j}$ live. For the homogeneous eight-vertex model and the XYZ chain we have $u_{1}=\ldots=u_{M}=0$. 
With these definitions the form factors are given by

$$
{ }^{(i)}\left\langle 0|\mathcal{O}| a_{1} \theta_{1}, \ldots, a_{N} \theta_{N}\right\rangle^{(i)}=\frac{1}{\chi} \operatorname{Tr}_{\mathcal{H}_{i}}\left(\Psi_{a_{N}}^{*}\left(v_{N}\right) \ldots \Psi_{a_{1}}^{*}\left(v_{1}\right) \hat{\mathcal{O}} \rho\right), \quad \chi=\chi\left(x^{4}\right) .
$$

Now consider the SOS model. Consider semi-infinite paths, $i$. e. the sequences of heights $n_{0}, n_{1}, n_{2}, \ldots$, such that $\left|n_{k}-n_{k+1}\right|=1$. Let $\mathcal{H}_{m n}$ be the space of formal linear combinations of all semi-infinite paths such that $n_{0}=n$ and either $n_{2 k}=m, n_{2 k+1}=m+1$ or $n_{2 k}=m+1, n_{2 k+1}=m$ depending on the parity of $m-n$ for large enough $k$. Introduce the operators

$$
\rho_{m n}=[n] x^{4 H_{m n}}: \mathcal{H}_{m n} \rightarrow \mathcal{H}_{m n}, \quad \Phi(u)_{m n}^{m n^{\prime}}: \mathcal{H}_{m n} \rightarrow \mathcal{H}_{m n^{\prime}}, \quad \Psi^{*}(u)_{m n}^{m^{\prime} n}: \mathcal{H}_{m n} \rightarrow \mathcal{H}_{m^{\prime} n}
$$

Here $m^{\prime}=m \pm 1, n^{\prime}=n \pm 1, i$. e. the pairs $\left(m^{\prime}, m\right)$ and $\left(n^{\prime}, n\right)$ are admissible. Below we always write simply $\Phi(u)_{n}^{n^{\prime}}$ and $\Psi^{*}(u)_{m}^{m^{\prime}}$. The commutation relations read

$$
\begin{gathered}
\Phi\left(u_{1}\right)_{s}^{n^{\prime}} \Phi\left(u_{2}\right)_{n}^{s}=\sum_{s^{\prime}} W\left[\begin{array}{cc}
n^{\prime} & s^{\prime} \\
s & n
\end{array} \mid u_{1}-u_{2}\right] \Phi\left(u_{2}\right)_{s^{\prime}}^{n^{\prime}} \Phi\left(u_{1}\right)_{n}^{s^{\prime}}, \\
\sum_{s^{\prime}} \tilde{W}\left[\begin{array}{cc}
m^{\prime} & s \\
s^{\prime} & m
\end{array} \mid u_{1}-u_{2}\right] \Psi^{*}\left(u_{1}\right)_{s^{\prime}}^{m^{\prime}} \Psi^{*}\left(u_{2}\right)_{m}^{s^{\prime}}=\Psi^{*}\left(u_{2}\right)_{s}^{m^{\prime}} \Psi^{*}\left(u_{1}\right)_{m}^{s}, \\
\Phi\left(u_{1}\right)_{n}^{n^{\prime}} \Psi^{*}\left(u_{2}\right)_{m}^{m^{\prime}}=\tau\left(u_{1}-u_{2}\right) \Psi^{*}\left(u_{2}\right)_{m}^{m^{\prime}} \Phi\left(u_{1}\right)_{n}^{n^{\prime}}
\end{gathered}
$$

and

$$
\Phi(u)_{n}^{n^{\prime}} \frac{\rho_{m n}}{[n]}=\frac{\rho_{m n^{\prime}}}{\left[n^{\prime}\right]} \Phi(u-2)_{n}^{n^{\prime}}, \quad \Psi^{*}(u)_{m}^{m^{\prime}} \rho_{m n}=\rho_{m^{\prime} n} \Psi^{*}(u-2)_{m}^{m^{\prime}}
$$

Here

$$
\tilde{W}\left[\begin{array}{ll}
m_{4} & m_{3} \\
m_{1} & m_{2}
\end{array} \mid u\right]=-W\left[\begin{array}{cc}
m_{4} & m_{3} \\
m_{1} & m_{2}
\end{array} \mid u ; \epsilon, r-1\right]
$$

The normalization conditions look like

$$
\begin{gathered}
\sum_{n^{\prime}} \Phi^{*}(u)_{n^{\prime}}^{n} \Phi(u)_{n}^{n^{\prime}}=1, \quad \Phi(u)_{n}^{n^{\prime \prime}} \Phi^{*}(u)_{n^{\prime}}^{n}=\delta_{n^{\prime \prime} n^{\prime}}, \\
\Psi\left(u^{\prime}\right)_{m}^{m^{\prime \prime}} \Psi^{*}(u)_{m^{\prime}}^{m}=\frac{1}{\pi} \frac{\delta_{m^{\prime \prime} m^{\prime}}}{u^{\prime}-u}+O\left(\left(u^{\prime}-u\right)^{0}\right)
\end{gathered}
$$

(admissibility of the pairs $\left(n, n^{\prime}\right),\left(n, n^{\prime \prime}\right),\left(m, m^{\prime}\right)$, and $\left(m, m^{\prime \prime}\right)$ is assumed) with

$$
\Phi^{*}(u)_{n}^{n^{\prime}}=(-)^{n-m_{0}+1}\left(n^{\prime}-n\right)[n] \Phi(u-1)_{n}^{n^{\prime}}, \quad \Psi(u)_{m}^{m^{\prime}}=(-)^{m-m_{0}+1} \frac{m^{\prime}-m}{[m]^{\prime}} \Psi^{*}(u-1)_{m}^{m^{\prime}} .
$$

Here

$$
[u]_{i}^{\prime}=h_{i}(u ; r-1)
$$

We want to incorporate the vertex-face correspondence into the vertex operator algebra. For the type I vertex operators it was done in [1]. The result can be formulated as follows. There exist two operators

$$
T\left(u_{0}\right)_{m n}: \mathcal{H}_{m n} \rightarrow \mathcal{H}_{i}, \quad T\left(u_{0}\right)^{m n}: \mathcal{H}_{i} \rightarrow \mathcal{H}_{m n}, \quad i=n-m \quad(\bmod 2),
$$

such that

$$
[m]^{\prime} \rho^{(i)}=\sum_{n \in 2 \mathbf{Z}+m+i} T\left(u_{0}\right)_{m n} \rho_{m n} T\left(u_{0}\right)^{m n}
$$

and

$$
\begin{aligned}
& \sum_{\varepsilon} t_{\varepsilon}^{*}\left(u-u_{0}\right)_{n^{\prime}}^{n} \Phi_{\varepsilon}(u) T\left(u_{0}\right)_{m n}=T\left(u_{0}\right)_{m n^{\prime}} \Phi(u)_{n}^{n^{\prime}}, \\
& \sum_{n} t_{\varepsilon}\left(u-u_{0}\right)_{n}^{n^{\prime}} \Phi(u)_{n}^{n^{\prime}} T\left(u_{0}\right)^{m n}=T\left(u_{0}\right)^{m n^{\prime}} \Phi_{\varepsilon}(u)
\end{aligned}
$$




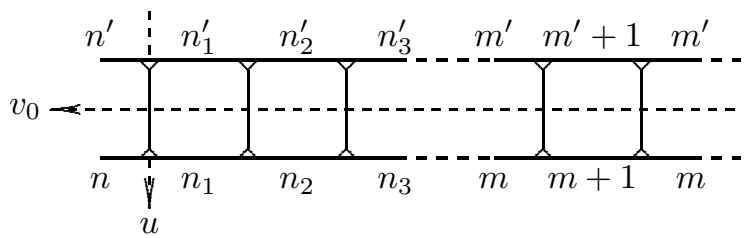

Fig. 5. The operator $\Lambda\left(u_{0}\right)_{m}^{m^{\prime}} \underset{n}{n^{\prime}}\left(u_{0}=u-v_{0}\right)$. The right solid part represents the condition at the infinity that corresponds to the ground state sector $m$. The upper bold line forms the operator $T\left(u_{0}\right)^{m^{\prime} n^{\prime}}$. The lower one forms $T\left(u_{0}\right)_{m n}$.

or, equivalently,

$$
\begin{aligned}
\Phi_{\varepsilon}(u) T\left(u_{0}\right)_{m n} & =\sum_{n^{\prime}} t_{\varepsilon}^{\prime}\left(u-u_{0}\right)_{n}^{n^{\prime}} T\left(u_{0}\right)_{m n^{\prime}} \Phi(u)_{n}^{n^{\prime}}, \\
\Phi(u)_{n}^{n^{\prime}} T\left(u_{0}\right)^{m n} & =\sum_{\varepsilon} t_{\varepsilon}^{*}\left(u-u_{0}\right)_{n^{\prime}}^{n} T\left(u_{0}\right)^{m n^{\prime}} \Phi_{\varepsilon}(u) .
\end{aligned}
$$

It is straightforward to prove that (3.17), (3.18a) are consistent with the algebras of $\Phi$ and $\rho$ operators for the eight-vertex and SOS models (see Appendix A).

As our aim is to express the objects of the eight-vertex model in terms of the objects in the SOS model, which are considered to be well defined due to the Lukyanov-Pugai free field representation, let us look at what these relations mean for the SOS model. Consider the operator

$$
\Lambda\left(u_{0}\right)_{m}^{m_{n}^{\prime} n^{\prime}}=T\left(u_{0}\right)^{m^{\prime} n^{\prime}} T\left(u_{0}\right)_{m n}: \mathcal{H}_{m n} \rightarrow \mathcal{H}_{m n}
$$

In the case of vacuum expectation values, which correspond to $N=0$ in (3.9), one can pull e.g. $T\left(u_{0}\right)^{m n}$ to the right by use of the cyclic property of trace and express $\langle\mathcal{O}\rangle$ in terms of traces over $\mathcal{H}_{m n}$ of operators $\Phi\left(u_{j}\right)_{n}^{n^{\prime}}, \rho_{m n}$, and $\Lambda\left(u_{0}\right)_{m n}^{m n^{\prime}}$ :

$$
\begin{aligned}
\langle\mathcal{O}\rangle^{(i)}= & \sum_{n \in \mathbf{Z}+m+i} \sum_{\left\{n_{k}\right\},\left\{\nu_{k}\right\}, n^{\prime}} t_{\varepsilon_{M}^{\prime}}^{*}\left(u_{M}-u_{0}\right)_{\nu_{M-1}}^{n} \ldots t_{\varepsilon_{1}^{\prime}}^{*}\left(u_{1}-u_{0}\right)_{n_{0}}^{\nu_{1}} t_{\varepsilon_{1}}\left(u_{1}-u_{0}\right)_{n_{1}}^{n_{0}} \ldots t_{\varepsilon_{M}}\left(u_{M}-u_{0}\right)_{n^{\prime}-1}^{n_{M-1}} \\
& \times \frac{1}{\chi} \operatorname{Tr}_{\mathcal{H}_{m n}}\left(\Phi^{*}\left(u_{M}\right)_{\nu_{M-1}}^{n} \ldots \Phi^{*}\left(u_{1}\right)_{n_{0}}^{\nu_{1}} \Phi\left(u_{1}\right)_{n_{1}}^{n_{0}} \ldots \Phi\left(u_{M}\right)_{n^{\prime}}^{n_{M-1}} \Lambda\left(u_{0}\right)_{m n}^{m n^{\prime}} \frac{\rho_{m n}}{[m]^{\prime}}\right)
\end{aligned}
$$

The operator $\Lambda$ is an infinite tail of the intertwining vectors that cannot be cancelled if something nonintegrable is inserted into a finite part of the lattice (Fig. 5).

From (3.18a) we easily obtain

$$
\Lambda\left(u_{0}\right)_{m s}^{m^{\prime} n^{\prime}} \Phi(u)_{n}^{s}=\sum_{s^{\prime}} L\left[\begin{array}{cc}
n^{\prime} & s^{\prime} \\
s & n
\end{array} \mid u_{0}-u\right] \Phi(u)_{s^{\prime}}^{n^{\prime}} \Lambda\left(u_{0}\right)_{m}^{m^{\prime} s^{\prime}}
$$

with

$$
L\left[\begin{array}{ll}
n_{4} & n_{3} \\
n_{1} & n_{2}
\end{array} \mid u\right]=\sum_{\varepsilon} t_{\varepsilon}^{*}(-u)_{n_{1}}^{n_{2}} t_{\varepsilon}(-u)_{n_{3}}^{n_{4}}
$$

Explicitly,

$$
\begin{aligned}
& L\left[\begin{array}{cc}
n^{\prime} & n^{\prime} \pm 1 \\
n & n \pm 1
\end{array} \mid u\right]=\frac{\left[u \mp \frac{1}{2}\left(n-n^{\prime}\right)\right]\left[\frac{1}{2}\left(n+n^{\prime}\right)\right]}{[u][n]}, \\
& L\left[\begin{array}{cc}
n^{\prime} & n^{\prime} \mp 1 \\
n & n \pm 1
\end{array} \mid u\right]=\frac{\left[u \mp \frac{1}{2}\left(n+n^{\prime}\right)\right]\left[\frac{1}{2}\left(n-n^{\prime}\right)\right]}{[u][n]}
\end{aligned}
$$

for $n^{\prime}-n \in 2 \mathbf{Z}$. Evidently,

$$
L\left[\begin{array}{cc}
n & n^{\prime \prime} \\
n & n^{\prime}
\end{array} \mid u\right]=\delta_{n^{\prime} n^{\prime \prime}}
$$


An important property of $\Lambda\left(u_{0}\right)$ is

$$
\Lambda\left(u_{0}\right)_{m n}^{m^{\prime} n}=\delta_{m^{\prime} m}
$$

It follows directly from Fig. 5 and Fig. 3b. It means that the $\Lambda$ operator does not appear in the case $\mathcal{O}=1$. Moreover,

$$
\Lambda\left(u_{0}\right)_{m}^{m^{\prime} n_{n}^{\prime}}=0 \quad \text { if } \quad m^{\prime}<m, n^{\prime}>n \text { or } m^{\prime}>m, n^{\prime}<n .
$$

Indeed, if $\Lambda$ is nonzero for, e. g., $n^{\prime}>n$ and $m^{\prime}<m$, there must exist a point $j$ on the half-infinite chain where $n_{j}^{\prime}=n_{j}$ and therefore $n_{k}^{\prime}=n_{k}$ for all $k>j$. Hence, $m^{\prime}=m$ which contradicts to the initial assumption.

Our next task is to extend the algebra (3.17), (3.18a) to the $\Psi$ operators. Let $\tilde{t}_{\varepsilon}(u)_{m}^{m^{\prime}}$ be, up to a scalar factor, $t_{\varepsilon}(u)_{m}^{m^{\prime}}$ after substitution $r \rightarrow r^{\prime}=r-1$. More precisely,

$$
\tilde{t}_{+}(u)_{m}^{m^{\prime}}=\tilde{f}(u) \theta_{3}\left(\frac{\left(m^{\prime}-m\right) u+m^{\prime}}{2 r^{\prime}} ; \mathrm{i} \frac{\pi}{2 \epsilon r^{\prime}}\right), \quad \tilde{t}_{-}(u)_{m}^{m^{\prime}}=(-1)^{m-m_{0}+1} \tilde{f}(u) \theta_{4}\left(\frac{\left(m^{\prime}-m\right) u+m^{\prime}}{2 r^{\prime}} ; \mathrm{i} \frac{\pi}{2 \epsilon r^{\prime}}\right)
$$

with $\tilde{f}(u)$ satisfying the equation

$$
[u]^{\prime} \tilde{f}(u) \tilde{f}(u-1)=C^{\prime} \equiv \frac{[0]_{4}^{\prime 2}}{2 \theta_{3}\left(0 ; \mathrm{i} \pi / 2 \epsilon r^{\prime}\right) \theta_{4}\left(0 ; \mathrm{i} \pi / 2 \epsilon r^{\prime}\right)} .
$$

This equation has an infinite number of solutions. The relevant solution of this equation will be chosen in Sec. 5 after discussing the free field representation and an explicit calculation of a form factor in a particular case. Here we cite the answer:

$$
\begin{aligned}
\tilde{f}(u) & =\mathrm{i} \sqrt{C^{\prime}} x^{-u^{2} / 2(r-1)-r u / 2(r-1)-1 / 4} \tilde{f}_{1}\left(x^{2 u}\right), \\
\tilde{f}_{1}(z) & =\frac{1}{\sqrt{\left(x^{2 r-2} ; x^{2 r-2}\right)_{\infty}}} \frac{\left(x^{2 r+2} z ; x^{4}, x^{2 r-2}\right)_{\infty}\left(x^{2} z^{-1} ; x^{4}, x^{2 r-2}\right)_{\infty}}{\left(x^{2 r} z ; x^{4}, x^{2 r-2}\right)_{\infty}\left(z^{-1} ; x^{4}, x^{2 r-2}\right)_{\infty}} .
\end{aligned}
$$

Consider the algebra

$$
\begin{aligned}
\Psi_{\varepsilon}^{*}(u) T_{m n}\left(u_{0}\right) & =\sum_{m^{\prime}} T_{m^{\prime} n}\left(u_{0}\right) \Psi^{*}(u)_{m}^{m^{\prime}} \tilde{t}_{\varepsilon}^{*}\left(u-u_{0}-\Delta u_{0}\right)_{m^{\prime}}^{m} \\
\Psi^{*}(u)_{m}^{m^{\prime}} T^{m n}\left(u_{0}\right) & =\sum_{\varepsilon} T^{m^{\prime} n}\left(u_{0}\right) \Psi_{\varepsilon}^{*}(u) \tilde{t}_{\varepsilon}\left(u-u_{0}-\Delta u_{0}\right)_{m}^{m^{\prime}}
\end{aligned}
$$

or, equivalently,

$$
\begin{gathered}
\sum_{\varepsilon} \Psi_{\varepsilon}^{*}(u) T_{m n}\left(u_{0}\right) \tilde{t}_{\varepsilon}\left(u-u_{0}-\Delta u_{0}\right)_{m}^{m^{\prime}}=T_{m^{\prime} n}\left(u_{0}\right) \Psi^{*}(u)_{m}^{m^{\prime}}, \\
\sum_{m} \Psi^{*}(u)_{m}^{m^{\prime}} T^{m n}\left(u_{0}\right) \tilde{t}_{\varepsilon}^{*}\left(u-u_{0}-\Delta u_{0}\right)_{m^{\prime}}^{m}=T^{m^{\prime} n}\left(u_{0}\right) \Psi_{\varepsilon}^{*}(u) .
\end{gathered}
$$

This algebra is consistent with (3.3-7), (3.11-13), (3.18a) for any value of $\Delta u_{0}$ subject to (3.17) (see Appendix A). The value of $\Delta u_{0}$ will be fixed by the free field representation in Sec. 4. Now rewrite these equations in terms of $\Lambda\left(u_{0}\right)$ :

$$
\Psi^{*}(u)_{s}^{m^{\prime}} \Lambda\left(u_{0}\right)_{m n}^{s} n^{\prime}=\sum_{s^{\prime}} \Lambda\left(u_{0}\right)_{s^{\prime}}^{m^{\prime} n_{n}^{\prime}} \Psi^{*}(u)_{m}^{s^{\prime}} \tilde{L}\left[\begin{array}{cc}
m^{\prime} & s \\
s^{\prime} & m
\end{array} \mid u_{0}+\Delta u_{0}-u\right],
$$

where

$$
\tilde{L}\left[\begin{array}{ll}
m_{4} & m_{3} \\
m_{1} & m_{2}
\end{array} \mid u\right]=\sum_{\varepsilon} \tilde{t}_{\varepsilon}^{*}(-u)_{m_{1}}^{m_{2}} \tilde{t}_{\varepsilon}(-u)_{m_{3}}^{m_{4}}=L\left[\begin{array}{cc}
m_{4} & m_{3} \\
m_{1} & m_{2}
\end{array} \mid u ; \epsilon, r-1\right] .
$$

To stress the difference between commutation relations (3.21a) and (3.21b) let us write down the simplest of them explicitly. For $n^{\prime}=s$ in (3.21a) using (3.24) we obtain the identity

$$
\Lambda\left(u_{0}\right)_{m}^{m^{\prime} n_{n^{\prime}}^{\prime}} \Phi(u)_{n}^{n^{\prime}}=\Phi(u)_{n}^{n^{\prime}} \Lambda\left(u_{0}\right)_{m}^{m_{n}^{\prime} n}
$$


which is trivial due to (3.25). Now let $n^{\prime}<n$. For $s=m$ in (3.21b) we have

$$
\begin{aligned}
\Psi^{*}(u)_{m}^{m+1} \Lambda\left(u_{0}\right)_{m n}^{m n^{\prime}}= & \Lambda\left(u_{0}\right)_{m+1 n}^{m+1 n^{\prime}} \Psi^{*}(u)_{m}^{m+1} \\
\Psi^{*}(u)_{m}^{m-1} \Lambda\left(u_{0}\right)_{m n}^{m n^{\prime}}= & \Lambda\left(u_{0}\right)_{m-1 n}^{m-1 n^{\prime}} \Psi^{*}(u)_{m}^{m-1} \\
& +\Lambda\left(u_{0}\right)_{m+1 n}^{m-1 n^{\prime}} \Psi^{*}(u)_{m}^{m+1} \frac{\left[u_{0}+\Delta u_{0}-u+m\right]^{\prime}[1]^{\prime}}{\left[u_{0}+\Delta u_{0}-u\right]^{\prime}[m+1]^{\prime}}
\end{aligned}
$$

These simplest commutation relations turn out to be nontrivial. They mean that the diagonal in $m$ elements of $\Lambda$ commute with one component of $\Psi^{*}(u)$, while the commutator with the second one contains an offdiagonal in $m$ matrix element of $\Lambda$. Below, the identity (3.30b) will give us a hint how to find the free field realization of the off-diagonal elements of the $\Lambda$ operator.

\section{Free field representation}

Recall the free field representation of the SOS model [6]. Consider a Heisenberg algebra of operators $a_{k}$ with nonzero integer $k$ and a pair of 'zero-mode' operators $\mathcal{P}$ and $\mathcal{Q}$ with the commutation relations

$$
[\mathcal{P}, \mathcal{Q}]=-\mathrm{i}, \quad\left[a_{k}, a_{l}\right]=k \frac{\llbracket k \rrbracket_{x} \llbracket(r-1) k \rrbracket_{x}}{\llbracket 2 k \rrbracket_{x} \llbracket r k \rrbracket_{x}} \delta_{k+l, 0} \quad \text { with } \quad \llbracket u \rrbracket_{x}=\frac{x^{u}-x^{-u}}{x-x^{-1}} .
$$

The ' $q$-number' $\llbracket u \rrbracket_{x}$ here should not be confused with the 'elliptic $q$-numbers' $[u]_{i}(i=1, \ldots, 4)$. It is also useful to introduce the operators

$$
\tilde{a}_{k}=\frac{\llbracket r k \rrbracket_{x}}{\llbracket(r-1) k \rrbracket_{x}} a_{k} .
$$

The normal ordering operation $: \ldots$. : places $\mathcal{P}$ to the right of $\mathcal{Q}$ and $a_{k}$ with positive $k$ to the right of $a_{-k}$. It will be convenient to assign

$$
\alpha_{+}=\sqrt{a_{+}}=\sqrt{\frac{r}{r-1}}, \quad \alpha_{-}=-\sqrt{a_{-}}=-\sqrt{\frac{r-1}{r}}, \quad 2 \alpha_{0}=\alpha_{+}+\alpha_{-}=\frac{1}{\sqrt{r(r-1)}} .
$$

Now introduce the fields

$$
\begin{aligned}
& \varphi(z)=\frac{\alpha_{-}}{\sqrt{2}}(\mathcal{Q}-\mathrm{i} \mathcal{P} \log z)-\sum_{k \neq 0} \frac{a_{k}}{\mathrm{i} k} z^{-k} . \\
& \tilde{\varphi}(z)=\frac{\alpha_{+}}{\sqrt{2}}(\mathcal{Q}-\mathrm{i} \mathcal{P} \log z)+\sum_{k \neq 0} \frac{\tilde{a}_{k}}{\mathrm{i} k} z^{-k} .
\end{aligned}
$$

These fields enter the exponential operators

$$
\begin{aligned}
& V(u)=z^{(r-1) / 4 r}: \mathrm{e}^{\mathrm{i} \varphi(z)}:, \quad \bar{V}(u)=z^{(r-1) / r}: \mathrm{e}^{-\mathrm{i} \varphi\left(x^{-1} z\right)-\mathrm{i} \varphi(x z)}:, \\
& \tilde{V}(u)=z^{r / 4(r-1)}: \mathrm{e}^{\mathrm{i} \tilde{\varphi}(z)}:, \quad \tilde{\bar{V}}(u)=z^{r /(r-1)}: \mathrm{e}^{-\mathrm{i} \tilde{\varphi}\left(x^{-1} z\right)-\mathrm{i} \tilde{\varphi}(x z)}:,
\end{aligned}
$$

and Lukyanov's screening operators

$$
\begin{aligned}
& x(u, C)=\frac{\epsilon}{\eta} \int_{C} \frac{d v}{\mathrm{i} \pi} \bar{V}(v) \frac{\left[v-u+\frac{1}{2}-\sqrt{2 r(r-1)} \mathcal{P}\right]}{\left[v-u-\frac{1}{2}\right]}, \\
& \tilde{x}(u, C)=\frac{\epsilon}{\eta^{\prime}} \int_{C} \frac{d v}{\mathrm{i} \pi} \tilde{\bar{V}}(v) \frac{\left[v-u-\frac{1}{2}+\sqrt{2 r(r-1)} \mathcal{P}\right]^{\prime}}{\left[v-u+\frac{1}{2}\right]^{\prime}} .
\end{aligned}
$$

The constants $\eta, \eta^{\prime}$ are defined by the normalization conditions (3.13) together with the definitions (4.11) below and read

$$
\begin{aligned}
\eta^{-1} & =\mathrm{i}[1] x^{\frac{r-1}{2 r}} \frac{\left(x^{2} ; x^{2 r}\right)_{\infty}}{\left(x^{2 r-2} ; x^{2 r}\right)_{\infty}} \frac{\left(x^{6} ; x^{4}, x^{2 r}\right)_{\infty}\left(x^{2 r+2} ; x^{4}, x^{2 r}\right)_{\infty}}{\left(x^{4} ; x^{4}, x^{2 r}\right)_{\infty}\left(x^{2 r+4} ; x^{4}, x^{2 r}\right)_{\infty}} \\
\eta^{-1} & =-\frac{2 \epsilon}{\pi}[1]^{\prime} x^{-\frac{r}{2(r-1)}} \frac{\left(x^{2 r-2} ; x^{2 r-2}\right)_{\infty}^{2}}{\left(x^{2 r} ; x^{2 r-2}\right)_{\infty}^{2}} \frac{\left(x^{4} ; x^{4}, x^{2 r-2}\right)_{\infty}\left(x^{2 r+2} ; x^{4}, x^{2 r-2}\right)_{\infty}}{\left(x^{2} ; x^{4}, x^{2 r-2}\right)_{\infty}\left(x^{2 r+4} ; x^{4}, x^{2 r-2}\right)_{\infty}}
\end{aligned}
$$


Now let us fix the contours. Let $C_{u}^{-}$and $C_{u}^{+}$go from $u-\frac{\mathrm{i} \pi}{2 \epsilon}$ to $u+\frac{\mathrm{i} \pi}{2 \epsilon}$ to the left and to the right of $u$ respectively. ${ }^{b}$ Then

$$
\begin{array}{ll}
X(u)=x\left(u, C_{u+1 / 2}^{-}\right), & Y(u)=x\left(u-1, C_{u-1 / 2}^{+}\right), \\
\tilde{X}(u)=\tilde{x}\left(u, C_{u-1 / 2}^{-}\right), & \tilde{Y}(u)=\tilde{x}\left(u+1, C_{u+1 / 2}^{+}\right) .
\end{array}
$$

These operators satisfy the equations

$$
Y(u) V(u)=V(u) X(u), \quad \tilde{Y}(u) \tilde{V}(u)=\tilde{V}(u) \tilde{X}(u) .
$$

Define the Fock spaces $\mathcal{F}_{m n}$ generated by the operators $a_{-k}(k>0)$ from the highest weight vectors $\left|P_{m n}\right\rangle$ such that

$$
a_{k}\left|P_{m n}\right\rangle=0 \quad(k>0), \quad \mathcal{P}\left|P_{m n}\right\rangle=P_{m n}\left|P_{m n}\right\rangle, \quad P_{m n}=\frac{1}{\sqrt{2}}\left(\alpha_{+} m+\alpha_{-} n\right) .
$$

There are strong evidences that $\mathcal{F}_{m n}$ can be identified with $\mathcal{H}_{m n}$ for generic $r$.

The vertex operators are defined on $\mathcal{F}_{m n}$ as follows:

$$
\begin{aligned}
\Phi(u)_{n}^{n+1} & =\frac{\mathrm{i}^{m-n}}{[n]} V(u), \\
\Phi(u)_{n}^{n-1} & =(-)^{n-m_{0}+1} \frac{\mathrm{i}^{m-n}}{[n]} V(u) X(u), \\
\Psi^{*}(u)_{m}^{m+1} & =\tilde{V}(u), \\
\Psi^{*}(u)_{m}^{m-1} & =(-)^{n-m_{0}} \tilde{Y}(u) \tilde{V}(u) .
\end{aligned}
$$

The corner Hamiltonian $H_{m n}$ is the restriction to $\mathcal{F}_{m n}$ of the operator

$$
H=\frac{\mathcal{P}^{2}}{2}+\sum_{k=1}^{\infty} \frac{\llbracket 2 k \rrbracket_{x} \llbracket r k \rrbracket_{x}}{\llbracket k \rrbracket_{x} \llbracket(r-1) k \rrbracket_{x}} a_{-k} a_{k}
$$

Later we shall need the following fact on the SOS model. The weights (2.5) are invariant under the substitution $n_{k} \rightarrow-n_{k}$. So the model is invariant under the change

$$
n_{k} \rightarrow-n_{k}, \quad m \rightarrow-m, \quad m_{0} \rightarrow-m_{0} .
$$

The free field representation (4.11) is not invariant under this substitution. So there exists another free field representation, where $\mathcal{H}_{m n}$ is identified with $\mathcal{F}_{-m-n}$ :

$$
\begin{aligned}
\Phi(u)_{n}^{n+1} & =(-)^{m-m_{0}} \frac{\mathrm{i}^{m-n}}{[n]} V(u) X(u), \\
\Phi(u)_{n}^{n-1} & =(-)^{m-n+1} \frac{\mathrm{i}^{m-n}}{[n]} V(u), \\
\Psi^{*}(u)_{m}^{m+1} & =(-)^{n-m_{0}} \tilde{Y}(u) \tilde{V}(u), \\
\Psi^{*}(u)_{m}^{m-1} & =\tilde{V}(u) .
\end{aligned}
$$

Of course, this representation gives the same multipoint local height probabilities as the first representation.

${ }^{b}$ We assume that the contours $C_{u}^{ \pm}$go to the left of all poles in the 'main rectangle' related to the operators that are to the right of the screening operator and to the right of all poles related to the operators placed to the left of the screening operators. The 'main rectangle' is understood as a rectangle with sides $r$ along the real axis and $\frac{\pi}{\epsilon}$ along the imaginary axis that contains all points $u_{i}, v_{i}$ etc. It is well defined for large enough $r$ and for points $u_{i}, v_{i}, \ldots$ close enough to each other. In the gereral case the operator products are considered as analytic continuation from this region. 
Our task is to realize the operator $\Lambda(u)$ in terms of free fields. The elements diagonal in $m$ are known [1]. For $n^{\prime}=n-2 l \leq n$ they read

$$
\left.\Lambda(u)_{m n}^{m n-2 l}\right|_{\mathcal{F}_{m n}}=(-)^{\left(n-m_{0}+1\right) l} \frac{[n-2 l]}{[n]} X^{l}(u) \quad \text { for } l \geq 0 .
$$

The elements with $n^{\prime}>n$ have no realization in this representation. However, the operator $\Lambda$ enters each trace once. Therefore, one can apply the second representation (4.14) to the traces that contain $\Lambda$ operators with $n^{\prime}>n$ so that

$$
\left.\Lambda(u)_{m n}^{m n+2 l}\right|_{\mathcal{F}_{-m-n}}=(-)^{\left(n-m_{0}+1\right) l} \frac{[n+2 l]}{[n]} X^{l}(u) \quad \text { for } l \geq 0
$$

Let us return to the equations (3.30). In the free field representation, the equation (3.30a) is an evident consequence of commutativity of $\tilde{V}(u)$ and $X\left(u_{0}\right)$. The equation (3.30b) is less trivial. For $n^{\prime}=n-2$ it can be written as follows

$$
\Lambda\left(u_{0}\right)_{m}^{m-2}{ }_{n}^{n-2} \tilde{V}(u)=-\frac{\left[u_{0}+\Delta u_{0}-u\right]^{\prime}[m]^{\prime}}{\left[u_{0}+\Delta u_{0}-u+m-1\right]^{\prime}[1]^{\prime}} \frac{[n-2]}{[n]}\left[\tilde{Y}(u), X\left(u_{0}\right)\right] \tilde{V}(u)
$$

It can be expected that the operator $\Lambda\left(u_{0}\right)_{m}^{m-2} \underset{n}{n-2}$ is more or less proportional to the commutator of $\tilde{Y}$ and $X$. This commutator is calculated in Appendix C. The answer is

$$
\begin{aligned}
{\left.\left[\tilde{Y}\left(u^{\prime}\right), X(u)\right]\right|_{\mathcal{F}_{m n}}=} & \frac{\epsilon}{\eta \eta^{\prime} \operatorname{sh} \epsilon}\left(\frac{[m-1]^{\prime}}{\partial[0]^{\prime}} \frac{\left[u^{\prime}-u+\frac{1}{2}-n\right]}{\left[u^{\prime}-u-\frac{1}{2}\right]} W_{+}\left(u^{\prime}\right)\right. \\
& \left.+\frac{[n-1]}{\partial[0]} \frac{\left[u^{\prime}-u+\frac{3}{2}-m\right]^{\prime}}{\left[u^{\prime}-u+\frac{1}{2}\right]^{\prime}} W_{-}(u)\right)
\end{aligned}
$$

Here $\partial[0]=d[u] /\left.d u\right|_{u=0}, \partial[0]^{\prime}=d[u]^{\prime} /\left.d u\right|_{u=0}$ and

$$
W_{+}(u)=W\left(u+\frac{r}{2}\right), \quad W_{-}(u)=W\left(u-\frac{r-1}{2}\right)
$$

with

$$
\begin{aligned}
& W(u)=z^{1 / r(r-1)}: \mathrm{e}^{\mathrm{i} \varphi_{0}(z)}: \\
& \varphi_{0}(z)=-2 \sqrt{2} \alpha_{0}(\mathcal{Q}-\mathrm{i} \mathcal{P} \log z)-\sum_{k \neq 0} \frac{\llbracket 2 k \rrbracket_{x}}{\llbracket(r-1) k \rrbracket_{x}} \frac{a_{k}}{\mathrm{i} k} z^{-k} .
\end{aligned}
$$

It follows from the identities of Appendix B that

$$
W_{+}(u) \tilde{V}(u)=0
$$

It means that the first term in (4.16) does not contribute to $\Lambda(u)_{m}^{m-2} \underset{n}{n-2}$, and the expression

$$
\Lambda(u)_{m}^{m-2}{ }_{n}^{n-2}=-\frac{\epsilon}{\eta \eta^{\prime} \operatorname{sh} \epsilon} \frac{[m]^{\prime}}{[1]^{\prime}} \frac{[n-1][n-2]}{\partial[0][n]} W_{-}(u)
$$

is consistent with (3.30) for

$$
\Delta u_{0}=-1 / 2
$$

Consider the limit $u^{\prime} \rightarrow u-\frac{1}{2}$ in $(4.16 \mathrm{a})$. It is singular. It can be seen that the product $X(u) \tilde{Y}\left(u^{\prime}\right)$ is regular in this limit, while the product $\tilde{Y}\left(u^{\prime}\right) X(u)$ has a pole because of pinching contours between poles. It means that

$$
\Lambda(u)_{m}^{m-2}{ }_{n}^{n-2} \sim \lim _{u^{\prime} \rightarrow u}\left[u^{\prime}-u\right]^{\prime} \tilde{Y}\left(u^{\prime}-\frac{1}{2}\right) X(u) .
$$


Generally, we conjecture that

$$
\Lambda\left(u_{0}\right)_{m}^{m-2 k}{ }_{n}^{n-2 l} \sim \lim _{u^{\prime} \rightarrow u}\left[u^{\prime}-u\right]^{\prime} \tilde{Y}^{k}\left(u^{\prime}-\frac{1}{2}\right) X^{l}(u) .
$$

It is shown in Appendix C that the r. h. s. is well-defined and given by

$$
\begin{aligned}
& \lim _{u^{\prime} \rightarrow u}\left[u^{\prime}-u\right]^{\prime} \tilde{Y}^{k}\left(u^{\prime}-\frac{1}{2}\right) X^{l}(u) \\
& =(-)^{k+l+1} \frac{\epsilon}{\eta \eta^{\prime} \operatorname{sh} \epsilon} \frac{[k]^{\prime}[m-k]^{\prime}}{[1]^{\prime}} \frac{[l][n-l]}{[1] \partial[0]} \tilde{X}^{k-1}\left(u-\frac{1}{2}\right) W_{-}(u) Y^{l-1}(u) .
\end{aligned}
$$

It is important that the contours in $\tilde{X}^{k-1}$ go to the left of all poles while the contours in $Y^{l-1}$ go to the right of all poles.

So we have

$$
\begin{aligned}
\left.\Lambda(u)_{m}^{m-2 k}{ }_{n}^{n-2 l}\right|_{\mathcal{F}_{m n}} & =C_{m}^{m-2 k}{ }_{n}^{n-2 l} \lim _{u^{\prime} \rightarrow u}\left[u^{\prime}-u\right]^{\prime} \tilde{Y}^{k}\left(u^{\prime}-\frac{1}{2}\right) X^{l}(u) \\
& =D_{m}^{m-2 k}{ }_{n}^{n-2 l} \tilde{X}^{k-1}\left(u-\frac{1}{2}\right) W_{-}(u) Y^{l-1}(u), \\
\left.\Lambda(u)_{m}^{m+2 k}{ }_{n}^{n+2 l}\right|_{\mathcal{F}_{-m-n}} & =C_{m}^{m+2 k}{ }_{n}^{n+2 l} \lim _{u^{\prime} \rightarrow u}\left[u^{\prime}-u\right]^{\prime} \tilde{Y}^{k}\left(u^{\prime}-\frac{1}{2}\right) X^{l}(u) \\
& =D_{m}^{m+2 k}{ }_{n}^{n+2 l} \tilde{X}^{k-1}\left(u-\frac{1}{2}\right) W_{-}(u) Y^{l-1}(u),
\end{aligned}
$$

for $k, l>1$, with some coefficients $C_{m}^{m^{\prime}}{ }_{n}^{n^{\prime}}$ and $D_{m}^{m^{\prime}}{ }_{n}^{n^{\prime}}$. To find the coefficients let us turn to the commutation relations (3.21b). Their explicit form for $\Delta u_{0}=-1 / 2$ is written out in Appendix D. It is easy to find from them that

$$
\begin{gathered}
\Psi^{*}(u)_{m-2 k}^{m-2 k+1} \Lambda\left(u_{0}\right)_{m}^{m-2 k}{ }_{n}^{n-2 l}-\frac{\left[u_{0}-u-m+k-\frac{1}{2}\right]^{\prime}[k-1]^{\prime}}{\left[u_{0}-u-k+\frac{1}{2}\right]^{\prime}[m-k]^{\prime}} \Psi^{*}(u)_{m-2 k+2}^{m-2 k+1} \Lambda\left(u_{0}\right)_{m}^{m-2 k+2}{ }_{n}^{n-2 l} \\
=\frac{\left[u_{0}-u+\frac{1}{2}\right]^{\prime}[m]^{\prime}[m-2 k+1]^{\prime}}{\left[u_{0}-u-k+\frac{1}{2}\right]^{\prime}[m+1]^{\prime}[m-k]^{\prime}} \Lambda\left(u_{0}\right)_{m+1}^{m-2 k+1} \underset{n}{n-2 l} \Psi^{*}(u)_{m}^{m+1} .
\end{gathered}
$$

Note that $\left.\tilde{V}(u) W_{(} u_{0}\right)$ has a zero at $u=u_{0}+\frac{1}{2}$ and a pole at $u=u_{0}-\frac{1}{2}$. Hence, both sides of the equation vanish at $u=u_{0}+\frac{1}{2}$ and this point is not interesting. But at $u=u_{0}-\frac{1}{2}$ both terms of the $\mathrm{l}$. h. s. have a pole while the r. h. s. remains finite. It means that near this point we may write

$$
\Psi^{*}(u)_{m-2 k}^{m-2 k+1} \Lambda\left(u_{0}\right)_{m}^{m-2 k} \underset{n}{n-2 l}=\Psi^{*}(u)_{m-2 k+2}^{m-2 k+1} \Lambda\left(u_{0}\right)_{m}^{m-2 k+2} \underset{n}{n-2 l}+O(1), \quad u \rightarrow u_{0}-\frac{1}{2} .
$$

By use of (4.11) and (4.9) we obtain

$$
\tilde{V}(u) \Lambda\left(u_{0}\right)_{m}^{m-2 k} \underset{n}{n-2 l}=(-)^{n-m_{0}} \tilde{V}(u) \tilde{X}\left(u_{0}-\frac{1}{2}\right) \Lambda\left(u_{0}\right)_{m}^{m-2 k+2} \underset{n}{n-2 l}+O(1), \quad u \rightarrow u_{0}-\frac{1}{2} .
$$

Here we used that the product $\tilde{X}(u) \Lambda\left(u_{0}\right)$ is regular at this point. Hence,

$$
\Lambda\left(u_{0}\right)_{m}^{m-2 k}{ }_{n}^{n-2 l}=(-)^{n-m_{0}} \tilde{X}\left(u_{0}-\frac{1}{2}\right) \Lambda\left(u_{0}\right)_{m}^{m-2 k+2} \underset{n}{n-2 l} .
$$

Similarly, from (3.21a) and the identity $V(u) W_{-}(u)=0$ we obtain

$$
\Lambda\left(u_{0}\right)_{m}^{m-2 k}{ }_{n}^{n-2 l}=(-)^{n-m_{0}} \frac{[l][n][n-l]}{[l-1][n-2][n-l-1]} \Lambda\left(u_{0}\right)_{m}^{m-2 k} \underset{n-2}{n-2 l} Y\left(u_{0}\right) .
$$

Together with (4.18) it defines the $D$ and, therefore, $C$ coeffitients

$$
\begin{aligned}
D_{m}^{m-2 k}{ }_{n}^{n-2 l} & =(-)^{\left(n-m_{0}\right)(k+l)+1} \frac{\epsilon}{\eta \eta^{\prime} \operatorname{sh} \epsilon} \frac{[m]^{\prime}}{[1]^{\prime}} \frac{[l][n-l][n-2 l]}{\partial[0][1][n]} . \\
C_{m}^{m-2 k}{ }_{n}^{n-2 l} & =(-)^{\left(n-m_{0}+1\right)(k+l)} \frac{[m]^{\prime}}{[k]^{\prime}[m-k]^{\prime}} \frac{[n-2 l]}{[n]} .
\end{aligned}
$$


These expressions are valid for $k, l<0$ as well. Indeed, for $m^{\prime}>m, n^{\prime}>n$ we have $e . g . D_{m}^{m^{\prime} n^{\prime}}=$ $\left.D_{-m-n}^{-m^{\prime}-n^{\prime}}\right|_{m_{0} \rightarrow-m_{0}}$ by definition of the second representation. It is evident that $D$ coefficients defined by (4.24) satisfy this equation.

From (3.25) and (3.26) we know that $\Lambda\left(u_{0}\right)_{m}^{m \pm 2 k} \underset{n}{n}=\Lambda\left(u_{0}\right)_{m}^{m \pm 2 k} \underset{n}{n \mp 2 l}=0$ for $k, l>0$, while the elements $\Lambda\left(u_{0}\right)_{m}^{m}{ }_{n}^{n \pm 2 l}(l \geq 0)$ are given by (4.15). It means that we never encounter the situation when an element of the $\Lambda$ operator could not be realized in either the first or the second bosonic representation.

The formulas (4.23), (4.24) are the main result of the paper. To prove them one has to check the commutation relation $(3.21)$ with $\Lambda(u)$ from $(4.23)$. It is enough to check it for $(3.21 \mathrm{a})$. This is a more or less direct but rather cumbersome calculation, and basically the same as the proof of (3.25) by substituting it into (3.21a), given in the Appendix $\mathrm{C}$ of [1], so we only give guidelines of it in Appendix D. Eq. (3.21b) with $m^{\prime}<m$ follows directly from that with $m^{\prime}=m$, which was checked in [1], and (4.21).

The only thing that has not yet been fixed is the function $\tilde{f}(z)$ that enter the intertwining vectors for excitations. To fix it we need to evaluate, at least, the simplest form factor.

\section{Two-particle form factor of the $\sigma^{z}$ operator}

Consider the operator $\sigma^{z}$. Its free field realization is given by ${ }^{c}$

$$
\widehat{\sigma^{z}}=\sum_{\varepsilon} \varepsilon \Phi_{-\varepsilon}(u-1) \Phi_{\varepsilon}(u)
$$

It means that the two-particle form factor of this operator is given by

$$
F_{a_{1} a_{2}}^{(i)}\left(\theta_{1}, \theta_{2}\right) \equiv{ }^{(i)}\left\langle 0\left|\sigma^{z}\right| a_{1} \theta_{1}, a_{2} \theta_{2}\right\rangle=\frac{1}{\chi} \sum_{\varepsilon} \varepsilon \operatorname{Tr}_{\mathcal{H}_{i}}\left(\Psi_{a_{2}}^{*}\left(v_{2}\right) \Psi_{a_{1}}^{*}\left(v_{1}\right) \Phi_{-\varepsilon}(u-1) \Phi_{\varepsilon}(u) \rho\right)
$$

with $\theta_{j}=\pi^{2} / 2 \epsilon+\mathrm{i} \pi v_{j}$. The direct way to calculate it is to substitute (3.17) with arbitrary $m$ into the r. h. s. of (5.2) and using (3.18) to reduce the trace to a linear combination of the traces for the SOS model:

$$
F_{a_{1} a_{2}}^{(i)}\left(\theta_{1}, \theta_{2}\right)=\sum_{m_{1}, m_{2}} F_{m_{1} m_{2} m}^{(i)}\left(\theta_{1}, \theta_{2}\right) \tilde{t}_{a_{2}}^{*}\left(v_{2}-u_{0}+\frac{1}{2}\right)_{m_{1}}^{m} \tilde{t}_{a_{1}}^{*}\left(v_{1}-u_{0}+\frac{1}{2}\right)_{m_{2}}^{m_{1}}
$$

with

$$
\begin{aligned}
F_{m_{1} m_{2} m}^{(i)}\left(\theta_{1}, \theta_{2}\right) & \frac{1}{\chi} \sum_{\varepsilon} \sum_{n \in 2 \mathbf{Z}+m+i} \varepsilon \operatorname{Tr}_{\mathcal{H}_{m n}}\left(\Psi^{*}\left(v_{2}\right)_{m_{2}}^{m} \Psi^{*}\left(v_{1}\right)_{m_{1}}^{m_{2}} T\left(u_{0}\right)^{m_{2} n} \Phi_{-\varepsilon}(u-1) \Phi_{\varepsilon}(u) T\left(u_{0}\right)_{m n} \frac{\rho_{m n}}{[m]^{\prime}}\right) \\
= & \frac{1}{\chi} \sum_{\varepsilon} \sum_{n \in 2 \mathbf{Z}+m+i} \sum_{n_{1} n_{2}} \varepsilon t_{-\varepsilon}\left(u-u_{0}-1\right)_{n_{2}}^{n} t_{\varepsilon}\left(u-u_{0}\right)_{n_{1}}^{n_{2}} \\
& \times \operatorname{Tr}_{\mathcal{H}_{m n}}\left(\Psi^{*}\left(v_{2}\right)_{m_{2}}^{m} \Psi^{*}\left(v_{1}\right)_{m_{1}}^{m_{2}} \Phi(u-1)_{n_{2}}^{n} \Phi(u)_{n_{1}}^{n_{2}} \Lambda\left(u_{0}\right)_{m}^{m_{1}}{ }_{n}^{n_{1}} \frac{\rho_{m n}}{[m]^{\prime}}\right)
\end{aligned}
$$

Now we can apply the bosonization technique, but we prefer to use the following trick. By pulling $T\left(u_{0}\right)^{m_{1} n}$ in $(5.3)$ to the left by use of $(3.18 \mathrm{~b})$ we obtain

$$
F_{m_{1} m_{2} m}^{(i)}\left(\theta_{1}, \theta_{2}\right)=\sum_{a_{1} a_{2}} F_{a_{1} a_{2}}^{(i)}\left(\theta_{1}, \theta_{2}\right) \tilde{t}_{a_{2}}\left(v_{2}-u_{0}+\frac{1}{2}\right)_{m_{2}}^{m} \tilde{t}_{a_{1}}\left(v_{1}-u_{0}+\frac{1}{2}\right)_{m_{1}}^{m_{2}}
$$

The idea is to fix $m_{1}-m, m_{2}-m$ and to consider (5.5) as a functional equation for the form factor $F_{a_{1} a_{2}}^{(i)}\left(\theta_{1}, \theta_{2}\right)$ considered as an analytic function of variables $\theta_{1}, \theta_{2}, m$. The requirement of $m$-independence of $F_{a_{1} a_{2}}$ fixes the solution uniquely. Indeed, consider the products $\tilde{t}_{a_{1}}\left(v_{1}\right)_{m_{1}}^{m} \tilde{t}_{a_{2}}\left(v_{2}\right)_{m_{2}}^{m} /\left(a_{1} a_{2}\right)^{m-m_{0}} f\left(v_{1}\right) f\left(v_{2}\right)$

${ }^{c}$ Recall that $u=0$ for the homogeneous model. 
as theta-functions of the variable $w=m / 2 r^{\prime}$ with the quasiperiods 1 and $\mathrm{i} \pi / \epsilon r^{\prime}$. It is extracted from the periodicity properties that they belong to a four-dimensional space. Moreover, as $\tilde{t}_{+} / \tilde{t}_{-}$is not a constant in $w$, these products form a basis in this space for generic values of $v_{1}, v_{2}$.

Let us, for example, fix

$$
m_{2}+1=m_{1}+2=m .
$$

Then the only terms in (5.4) that make a nonzero contribution are those with

$$
n_{2}+1=n_{1}+2=n \text {. }
$$

It means that

$$
\begin{aligned}
F_{m-2 m-1 m}^{(i)}\left(\theta_{1}, \theta_{2}\right)= & \frac{1}{\chi} \sum_{\varepsilon} \sum_{n \in 2 \mathbf{Z}+m+i} \varepsilon t_{-\varepsilon}\left(u-u_{0}-1\right)_{n-1}^{n} t_{\varepsilon}\left(u-u_{0}\right)_{n-2}^{n-1} \\
& \times \operatorname{Tr}_{\mathcal{H}_{m n}}\left(\Psi^{*}\left(v_{2}\right)_{m-1}^{m} \Psi^{*}\left(v_{1}\right)_{m-2}^{m-1} \Phi(u-1)_{n-1}^{n} \Phi(u)_{n-2}^{n-1} \Lambda\left(u_{0}\right)_{m}^{m-2}{ }_{n}^{n-2} \frac{\rho_{m n}}{[m]^{\prime}}\right) .
\end{aligned}
$$

But $\Lambda\left(u_{0}\right)_{m}^{m-2}{ }_{n}^{n-2}$ is expressed in terms of the field $W_{-}\left(u_{0}\right)$ and contains no integrations. The $\Phi$ and $\Psi^{*}$ fields in this trace contain no integrations as well. It means that it is possible to obtain an explicit representation for the two-point form factor of $\sigma^{z}$ in terms of infinite products and elliptic functions only, without any integrations.

Let us make some remarks on traces of the vertex operators. Consider any operator $U(u)$ of the form

$$
U(u)=U^{0}(u) \mathrm{e}^{\phi^{+}(u)} \mathrm{e}^{\phi^{-}(u)},
$$

where $U^{0}(u)$ is the zero-mode operator of the form $z^{\alpha^{2} / 2} \mathrm{e}^{i \alpha \mathcal{Q}} z^{\alpha \mathcal{P}}$, and operators $\phi^{ \pm}(u)$ are positive/negative frequency parts of the form $\sum_{k=1}^{\infty} A_{ \pm k} a_{\mp k} z^{ \pm k}$.

Denote by $\operatorname{Tr}_{*}$ the trace over oscillator modes and by $H^{*}$ the oscillator contribution to $H_{m n}$. Besides, let

$$
\chi^{*}=\operatorname{Tr}_{*}\left(x^{4 H^{*}}\right)=\frac{1}{\left(x^{4} ; x^{4}\right)_{\infty}} .
$$

Then

$$
\operatorname{Tr}_{\mathcal{F}_{m n}}\left(U_{N}\left(u_{N}\right) \ldots U_{1}\left(u_{1}\right)\right)=\left\langle P_{m n}\left|U_{N}^{0}\left(u_{N}\right) \ldots U_{1}^{0}\left(u_{1}\right)\right| P_{m n}\right\rangle \chi^{*} \prod_{i=1}^{N} c_{i} \prod_{i<j} g_{i j}\left(u_{i}-u_{j}\right)
$$

with

$$
\begin{aligned}
\log c_{i} & =\frac{1}{\chi^{*}} \operatorname{Tr}_{*}\left(\phi_{i}^{+}(0) \phi_{i}^{-}(0) x^{4 H^{*}}\right), \\
\log g_{i j}(u) & =\frac{1}{\chi^{*}} \operatorname{Tr}_{*}\left(\phi_{i}(0) \phi_{j}(u) x^{4 H^{*}}\right), \quad \phi_{i}(u)=\phi_{i}^{+}(u)+\phi_{i}^{-}(u) .
\end{aligned}
$$

For the vertex operators used here the expressions for $c_{i}$ and $g_{i j}$ are listed in the Appendix E.

By substituting (4.11), (4.23a), and (4.24) into (5.6) we obtain

$$
\begin{aligned}
F_{m-2 m-1 m}^{(i)}\left(\theta_{1}, \theta_{2}\right) & =(-)^{m-m_{0}} \frac{c}{\chi} \sum_{n \in 2 \mathbf{Z}+m+i} K_{n} T_{m n}, \\
T_{m n} & =\operatorname{Tr}_{\mathcal{H}_{m n}}\left(\tilde{V}\left(v_{2}\right) \tilde{V}\left(v_{1}\right) V(u-1) V(u) W_{-}\left(u_{0}\right) x^{4 H}\right),
\end{aligned}
$$

where $c=\mathrm{i} \epsilon /\left([1]^{\prime} \partial[0] \eta \eta^{\prime} \operatorname{sh} \epsilon\right)$ and

$$
K_{n}=(-)^{n-m_{0}} \sum_{\varepsilon} \varepsilon t_{-\varepsilon}\left(u-u_{0}-1\right)_{n-1}^{n} t_{\varepsilon}\left(u-u_{0}\right)_{n-2}^{n-1}=\frac{\left[n-1+u-u_{0}\right]_{4}[0]_{4}}{\left[u-u_{0}\right]_{1}} .
$$

The trace $T_{m n}$ can be factorized as

$$
T_{m n}=h_{m n}\left(u, u_{0} ; v_{1}, v_{2}\right) g\left(u, u_{0} ; v_{1}, v_{2}\right) .
$$


Here $h_{m n}$ is the zero-mode contribution:

$$
\begin{aligned}
h_{m n}\left(u, u_{0} ; v_{1}, v_{2}\right)= & \left\langle P_{m n}\left|\tilde{V}^{0}\left(v_{2}\right) \tilde{V}^{0}\left(v_{1}\right) V^{0}(u-1) V^{0}(u) W_{-}^{0}\left(u_{0}\right) x^{2 \mathcal{P}^{2}}\right| P_{m n}\right\rangle \\
= & \mathrm{e}^{-\epsilon a_{+} m^{2}-2 \epsilon\left(a_{+}-2-\left(a_{+}-1\right) u_{0}-u+\frac{a_{+}}{2}\left(v_{1}+v_{2}\right)\right) m+\epsilon\left(2 a_{+}+a_{-}-1-2\left(2-a_{-}\right) u+\frac{a_{+}}{2}\left(3 v_{1}+v_{2}\right)\right)} \\
& \times \mathrm{e}^{-\epsilon a_{-} n^{2}+2 \epsilon\left(m+1+a_{-}-\left(1-a_{-}\right) u_{0}-a_{-} u+\frac{1}{2}\left(v_{1}+v_{2}\right)\right) n} .
\end{aligned}
$$

The function $g\left(u, u_{0} ; v_{1}, v_{2}\right)$ is the trace over the oscillators $a_{k}$ and is given by

$$
\begin{aligned}
g\left(u, u_{0} ; v_{1}, v_{2}\right)= & \chi^{*} c_{\tilde{V}}^{2} c_{V}^{2} c_{W} g_{V V}(1) g_{\tilde{V} \tilde{V}}\left(v_{1}-v_{2}\right) g_{V W_{-}}\left(u_{0}-u\right) g_{V W_{-}}\left(u_{0}-u+1\right) \\
& \times \prod_{j=1,2} g_{\tilde{V} V}\left(u-v_{j}\right) g_{\tilde{V} V}\left(u-v_{j}-1\right) g_{\tilde{V} W_{-}}\left(u_{0}-v_{j}\right) .
\end{aligned}
$$

The sum

$$
h_{m}^{(i)}=\sum_{n \in 2 \mathbf{Z}+m+i} K_{n} h_{m n}\left(u, u_{0} ; v_{1}, v_{2}\right)
$$

can be calculated by the tricks described in Appendix D of [1] and reads

$$
\begin{aligned}
h_{m}^{(i)}=\frac{[0]_{4}}{2\left[u-u_{0}\right]_{1}} \mathrm{e}^{\epsilon k\left(u, u_{0} ; v_{1}, v_{2}\right)}( & {\left[m+\frac{v_{1}+v_{2}}{2}-u_{0}\right]_{4}^{\prime}\left\{\frac{v_{1}+v_{2}}{2}-u\right\}_{4} } \\
& \left.+(-1)^{i}\left[m+\frac{v_{1}+v_{2}}{2}-u_{0}\right]_{1}^{\prime}\left\{\frac{v_{1}+v_{2}}{2}-u\right\}_{1}\right)
\end{aligned}
$$

with

$$
\{u\}_{i}=h_{i}(u ; 1)
$$

and

$k\left(u, u_{0} ; v_{1}, v_{2}\right)=\left(u-\frac{v_{1}+v_{2}}{2}-2\right)^{2}+\frac{r}{r-1} \frac{v_{1}-v_{2}-2}{2}-\frac{r-1}{2 r}-\frac{\left(u-u_{0}\right)^{2}}{r}+\frac{1}{r-1}\left(u_{0}-\frac{v_{1}+v_{2}}{2}-1\right)^{2}$.

From the identity

$$
\theta_{4}(u ; \tau) \theta_{3}(v ; \tau) \pm \theta_{3}(u ; \tau) \theta_{4}(v ; \tau)=\frac{2 \theta_{3}(0 ; \tau) \theta_{4}(0 ; \tau)}{\theta_{4}^{2}(0 ; 2 \tau)} \theta_{4,1}(u-v ; 2 \tau) \theta_{4,1}(u+v ; 2 \tau)
$$

we obtain

$$
\begin{aligned}
\frac{1}{C^{\prime}}\left[\frac{v_{2}-v_{1}+1}{2}\right]_{4,1}^{\prime}\left[m+\frac{v_{1}+v_{2}-1}{2}\right]_{4,1}^{\prime}= & \theta_{4}\left(\frac{v_{2}+m}{2 r^{\prime}} ; \frac{\mathrm{i} \pi}{2 \epsilon r^{\prime}}\right) \theta_{3}\left(\frac{v_{1}+m-1}{2 r^{\prime}} ; \frac{\mathrm{i} \pi}{2 \epsilon r^{\prime}}\right) \\
& \pm \theta_{3}\left(\frac{v_{2}+m}{2 r^{\prime}} ; \frac{\mathrm{i} \pi}{2 \epsilon r^{\prime}}\right) \theta_{4}\left(\frac{v_{1}+m-1}{2 r^{\prime}} ; \frac{\mathrm{i} \pi}{2 \epsilon r^{\prime}}\right)
\end{aligned}
$$

with $C^{\prime}$ given by (3.28). By substituting (5.17) into (5.16) and taking into account (3.27a) we obtain that the form factor $F_{m-2 m-1 m}^{(i)}$ have the desired structure:

$$
\begin{aligned}
F_{m-2 m-1 m}^{(i)}\left(\theta_{1}, \theta_{2}\right)= & (-)^{m-m_{0}} \frac{c}{\chi} h_{m}^{(i)}\left(u, u_{0} ; v_{1}, v_{2}\right) g\left(u, u_{0} ; v_{1}, v_{2}\right) \\
= & A_{m}^{(i)}\left(u, u_{0} ; v_{1}, v_{2}\right) \tilde{t}_{-}\left(v_{2}-u_{0}+\frac{1}{2}\right)_{m-1}^{m} \tilde{t}_{+}\left(v_{1}-u_{0}+\frac{1}{2}\right)_{m-2}^{m-1} \\
& +B_{m}^{(i)}\left(u, u_{0} ; v_{1}, v_{2}\right) \tilde{t}_{+}\left(v_{2}-u_{0}+\frac{1}{2}\right)_{m-1}^{m} \tilde{t}_{-}\left(v_{1}-u_{0}+\frac{1}{2}\right)_{m-2}^{m-1} .
\end{aligned}
$$

Here

$$
\begin{aligned}
A_{m}^{(i)} & =G\left(u, u_{0} ; v_{1}, v_{2}\right)\left((-1)^{i} a_{1}\left(u ; v_{1}, v_{2}\right)+a_{4}\left(u ; v_{1}, v_{2}\right)\right), \\
B_{m}^{(i)} & =G\left(u, u_{0} ; v_{1}, v_{2}\right)\left((-1)^{i} a_{1}\left(u ; v_{1}, v_{2}\right)-a_{4}\left(u ; v_{1}, v_{2}\right)\right), \\
G\left(u, u_{0} ; v_{1}, v_{2}\right) & =\mathrm{e}^{\epsilon k\left(u, u_{0} ; v_{1}, v_{2}\right)} \frac{c C^{\prime}[0]_{4}}{2 \chi\left[u-u_{0}\right]_{1}} \frac{g\left(u, u_{0} ; v_{1}, v_{2}\right)}{\tilde{f}\left(v_{1}-u_{0}+\frac{1}{2}\right) \tilde{f}\left(v_{2}-u_{0}+\frac{1}{2}\right)}
\end{aligned}
$$


with

$$
a_{i}\left(u ; v_{1}, v_{2}\right)=\frac{\left\{\frac{1}{2}\left(v_{1}+v_{2}\right)-u\right\}_{i}}{\left[\frac{1}{2}\left(v_{2}-v_{1}+1\right)\right]_{i}^{\prime}} .
$$

The factors $A_{m}^{(i)}$ and $B_{m}^{(i)}$ could be identified with $F_{+-}\left(\theta_{1}, \theta_{2}\right)$ and $F_{-+}\left(\theta_{1}, \theta_{2}\right)$ if we could prove that they are $u_{0}$ independent. To do it let us gather the $u_{0}$ dependent factors of these expressions:

$$
e^{\epsilon k\left(u, u_{0} ; v_{1}, v_{2}\right)} \frac{g_{V W_{-}}\left(u_{0}-u\right) g_{V W_{-}}\left(u_{0}-u+1\right) g_{\tilde{V} W_{-}}\left(u_{0}-v_{1}\right) g_{\tilde{V} W_{-}}\left(u_{0}-v_{2}\right)}{\left[u-u_{0}\right] \tilde{f}\left(v_{1}-u_{0}+\frac{1}{2}\right) \tilde{f}\left(v_{2}-u_{0}+\frac{1}{2}\right)} .
$$

Note that the product

$$
g_{V W_{-}}\left(u_{0}-u\right) g_{V W_{-}}\left(u_{0}-u+1\right)=-\mathrm{e}^{\epsilon\left(\left(u-u_{0}\right)^{2} / r+u-u_{0}\right)} \frac{\left[u-u_{0}\right]}{\left(x^{2 r} ; x^{2 r}\right)_{\infty}}
$$

in the numerator contains $\left[u-u_{0}\right]$ that cancels the same factor in the denuminator. So, up to an exponential factor, the function $\tilde{f}\left(v+\frac{1}{2}\right)$ must be proportional to $g_{\tilde{V} W_{-}}(-v)$. This uniquely determines the prefactor $\tilde{f}(u)$ in the form $(3.27 \mathrm{~b})$.

Finally, we obtain

$$
\begin{aligned}
F_{+-}^{(i)}\left(\theta_{1}, \theta_{2}\right)= & G\left(u ; v_{1}, v_{2}\right)\left((-1)^{i} a_{1}\left(u ; v_{1}, v_{2}\right)+a_{4}\left(u ; v_{1}, v_{2}\right)\right) \\
F_{-+}^{(i)}\left(\theta_{1}, \theta_{2}\right)= & G\left(u ; v_{1}, v_{2}\right)\left((-1)^{i} a_{1}\left(u ; v_{1}, v_{2}\right)-a_{4}\left(u ; v_{1}, v_{2}\right)\right), \\
G\left(u ; v_{1}, v_{2}\right)= & c c_{\tilde{V}}^{2} c_{V}^{2} c_{W} g_{V V}(1)[0]_{4} \frac{\chi^{*}}{2 \chi} \frac{\left(x^{2 r-2} ; x^{2 r-2}\right)_{\infty}}{\left(x^{2 r} ; x^{2 r}\right)_{\infty}} \mathrm{e}^{\epsilon k^{\prime}\left(u ; v_{1}, v_{2}\right)} \\
& \times g_{\tilde{V} \tilde{V}}\left(v_{1}-v_{2}\right) \prod_{j=1,2} g_{\tilde{V} V}\left(u-v_{j}\right) g_{\tilde{V} V}\left(u-v_{j}-1\right)
\end{aligned}
$$

with

$$
k^{\prime}\left(u ; v_{1}, v_{2}\right)=\left(u-\frac{v_{1}+v_{2}}{2}-\frac{3}{2}\right)^{2}+\frac{v_{1}-v_{2}-3 / 2}{2}-\frac{1}{r-1}\left(\frac{v_{1}-v_{2}-1}{2}\right)^{2}-\frac{1}{2 r(r-1)} .
$$

The other two components, $F_{++}$and $F_{--}$, of the form factor vanish in consistency with the charge conservation modulo 4 .

Now consider the limit $\theta_{2} \rightarrow \theta_{1}-\mathrm{i} \pi\left(\right.$ or $\left.v_{2} \rightarrow v_{1}-1\right)$. From (3.7) we obtain the equation

$$
F_{ \pm \mp}^{(i)}\left(\theta, \theta^{\prime}-\mathrm{i} \pi\right)=\frac{2 i}{\theta^{\prime}-\theta}\left\langle\sigma^{z}\right\rangle^{(i)}+O(1)
$$

which is a particular case of the Smirnov form factor axioms [13]. So, as a check of our construction we can calculate the expectation value of the spin operator at a given link in a given ground state sector. This reproduces the well-known Baxter-Kelland formula [14]

$$
\left\langle\sigma^{z}\right\rangle^{(i)}=(-)^{i} \frac{\left(x^{2} ; x^{2}\right)_{\infty}^{2}}{\left(-x^{2} ; x^{2}\right)_{\infty}^{2}} \frac{\left(-x^{2 r} ; x^{2 r}\right)_{\infty}^{2}}{\left(x^{2 r} ; x^{2 r}\right)_{\infty}^{2}}
$$

In contrast to [1] this formula was obtained here without any integrations.

\section{Discussion}

The main result of the paper is the bosonization recipe for the form factors consisting of the intertwining algebra for vertex operators $(3.16-18),(3.27),(4.19)$ and the bosonization prescription for the $\Lambda$ operator (4.23), (4.24). 
Of course, this recipe is based on several conjectures. We conjectured that the vertex operator algebras correctly describe the respective models, and that the bosonization procedure provides relevant representations of the vertex oprator algebra. Another problem is that it seems to be very difficult to obtain more or less simple general formulas for correlation functions and form factors, as it was done for the six-vertex model [3]. Moreover, the bosonization of the eight-vertex operators depends on two additional parameters $m$ and $u_{0}$, while the values of correlation functions and form factors are, of course, $m$ and $u_{0}$ independent. $^{d}$

In the case of the six-vertex/XXZ model both vertex operator approach and bosonization technique were based on the $U_{q}\left(\widehat{s l}_{2}\right)$ symmetry of the model [3] which was, in turn, based on the low-temperature expansions [12] and the theory of crystal bases [17]. The symmetry of the eight-vertex model was conjectured to be the elliptic algebra $\mathcal{A}_{q, p}\left(\widehat{s l}_{2}\right)$ [5], but neither low-temperature expansion check was performed nor bosonization of this algebra was acheived. The only encouraging result is its relation to the elliptic algebra $E_{\tau, \eta}\left(\widehat{s l}_{2}\right)$ describing the SOS model $[18,19]$. One might expect a natural solution of the problem of $m$ and $u_{0}$ independence in this framework.

Another approach to calculation of correlation functions of lattice models is based on the algebraic Bethe Ansatz method and seems to be more rigorous [20,21]. Though generalization of this approach to the eight-vertex model encounder significant difficulties, in principle, it could provide a more direct way to obtaining correlation functions and form factors.

An interesting feature of our construction is that two integrals, one of the $X$ type and the other of the $\tilde{X}$ type, in the form factors can be taken by use of the trick of Sec. 5. This feature shows promise of obtaining more explicit formulas for correlation functions and form factor. Note that the possibility of explicit taking two integrals is, probably, a general feature of form factors [22], though in the usual bosonization schemes it requires very sophisticated calculations.

\section{Acknowledgments}

The author is grateful to A. Belavin, S. Lukyanov, A. Odesskii, and Ya. Pugai for fruitful discussions and to M. Jimbo and T. Miwa for their hospitality at RIMS, Kyoto University in July, 1999, where many interesting discussions took place. The work was supported by INTAS under the grant INTAS-OPEN-97-1312, by CRDF under the grant RP1-2254 and by RFBR under the grants 99-01-01169 and 00-15-96579.

\section{Appendix A. Consistency of the vertex operator algebra}

Here we check consistency of the relations for the operators $T\left(u_{0}\right)_{m n}, T\left(u_{0}\right)^{m n}$ with the algebras of vertex operators for the eight-vertex and SOS models.

Consider the product

$$
T\left(u_{0}\right)_{m n^{\prime}} \Phi\left(u_{1}\right)_{s}^{n^{\prime}} \Phi\left(u_{2}\right)_{n}^{s}
$$

and try to reverse the order of the operators. One can do it in two ways. In the first way one can first push the operator $T\left(u_{0}\right)_{m n}$ through the vertex operators by use of (3.18), and then exchange the vertex operators by use of (3.3):

$$
\begin{aligned}
(A .1) & =\sum_{\varepsilon_{1} \varepsilon_{2}} t_{\varepsilon_{1}}\left(u_{1}-u_{0}\right)_{s}^{n^{\prime}} t_{\varepsilon_{2}}\left(u_{2}-u_{0}\right)_{n}^{s} \Phi_{\varepsilon_{1}}\left(u_{1}\right) \Phi_{\varepsilon_{2}}\left(u_{2}\right) T\left(u_{0}\right)_{m n} \\
& =\sum_{\varepsilon_{1} \varepsilon_{2} \varepsilon_{1}^{\prime} \varepsilon_{2}^{\prime}} t_{\varepsilon_{1}}\left(u_{1}-u_{0}\right)_{s}^{n^{\prime}} t_{\varepsilon_{2}}\left(u_{2}-u_{0}\right)_{n}^{s} R\left(u_{1}-u_{2}\right)_{\varepsilon_{1} \varepsilon_{2}}^{\varepsilon_{1}^{\prime} \varepsilon_{2}^{\prime}} \Phi_{\varepsilon_{2}^{\prime}}\left(u_{2}\right) \Phi_{\varepsilon_{1}^{\prime}}\left(u_{1}\right) T\left(u_{0}\right)_{m n} .
\end{aligned}
$$

In the second way one can first commute vertex operators according to (3.11) and then push $T\left(u_{0}\right)_{m n}$ by use of (3.18):

$$
\begin{aligned}
(A .1) & =\sum_{s^{\prime}} W\left[\begin{array}{rr}
n^{\prime} & s^{\prime} \\
s & n
\end{array} \mid u_{1}-u_{2}\right] T\left(u_{0}\right)_{m n^{\prime}} \Phi\left(u_{2}\right)_{s^{\prime}}^{n^{\prime}} \Phi\left(u_{1}\right)_{n}^{s^{\prime}} \\
& =\sum_{s^{\prime} \varepsilon_{1} \varepsilon_{2}} W\left[\begin{array}{rr}
n^{\prime} & s^{\prime} \\
s & n
\end{array} \mid u_{1}-u_{2}\right] t_{\varepsilon_{2}}\left(u_{2}-u_{0}\right)_{s^{\prime}}^{n^{\prime}} t_{\varepsilon_{1}}\left(u_{1}-u_{0}\right)_{n}^{s^{\prime}} \Phi_{\varepsilon_{2}^{\prime}}\left(u_{2}\right) \Phi_{\varepsilon_{1}^{\prime}}\left(u_{1}\right) T\left(u_{0}\right)_{m n} .
\end{aligned}
$$

\footnotetext{
$d$ To be more precise, the parameter $u_{0}$ becomes a physical parameter in a model with a boundary [16].
} 
The last lines of (A.2) and (A.3) coincide because of the vertex-face correspondence relation (2.12). Consistency with other commutation relations for vertex operators is checked in the same way.

Now let us check consistency of the relation between the shift operators (3.17) with commutation relations. We have

$$
\begin{aligned}
\rho \Phi_{\varepsilon}(u-2)= & \sum_{n \in 2 \mathbf{Z}+m+i} T\left(u_{0}\right)_{m n} \frac{\rho_{m n}}{[m]^{\prime}} T\left(u_{0}\right)^{m n} \Phi_{\varepsilon}(u-2) \\
= & \sum_{n \in 2 \mathbf{Z}+m+i} \sum_{n^{\prime}=n \pm 1} t_{\varepsilon}\left(u-u_{0}-2\right)_{n^{\prime}}^{n} T\left(u_{0}\right)_{m n} \frac{\rho_{m n}}{[m]^{\prime}} \Phi(u-2)_{n^{\prime}}^{n} T\left(u_{0}\right)^{m n^{\prime}} \\
= & \sum_{n \in 2 \mathbf{Z}+m+i} \sum_{n^{\prime}=n \pm 1} \frac{[n]}{\left[n^{\prime}\right]} t_{\varepsilon}\left(u-u_{0}-2\right)_{n^{\prime}}^{n} T\left(u_{0}\right)_{m n} \Phi(u)_{n^{\prime}}^{n} \frac{\rho_{m n^{\prime}}}{[m]^{\prime}} T\left(u_{0}\right)^{m n^{\prime}} \\
= & \sum_{n \in 2 \mathbf{Z}+m+i} \sum_{n^{\prime}=n \pm 1} t_{\varepsilon}^{\prime}\left(u-u_{0}\right)_{n^{\prime}}^{n} T\left(u_{0}\right)_{m n} \Phi(u)_{n^{\prime}}^{n} \frac{\rho_{m n^{\prime}}}{[m]^{\prime}} T\left(u_{0}\right)^{m n^{\prime}} \\
= & \sum_{n^{\prime} \in 2 \mathbf{Z}+m+1-i n=n^{\prime} \pm 1} \sum_{\varepsilon^{\prime}} t_{\varepsilon}^{\prime}\left(u-u_{0}\right)_{n^{\prime}}^{n} t_{\varepsilon^{\prime}}^{*}\left(u-u_{0}\right)_{n}^{n^{\prime}} \Phi_{\varepsilon^{\prime}}(u) T\left(u_{0}\right)_{m n^{\prime}} \frac{\rho_{m n^{\prime}}}{[m]^{\prime}} T\left(u_{0}\right)^{m n^{\prime}} \\
= & \sum_{n^{\prime} \in 2 \mathbf{Z}+m+1-i} \Phi_{\varepsilon}(u) T\left(u_{0}\right)_{m n^{\prime}} \frac{\rho_{m n^{\prime}}}{[m]^{\prime}} T\left(u_{0}\right)^{m n^{\prime}} \\
= & \Phi_{\varepsilon}(u) \rho .
\end{aligned}
$$

Here (2.14) and (2.13) were used. This check (in different notations) was one of the key results of [1].

The check for the $\Psi$ operators is slightly different because it essentially uses the $m$ independence of $\rho$. Let $v_{0}=u_{0}+\Delta u_{0}$. Then

$$
\begin{aligned}
\Psi_{\varepsilon}^{*}(u) \rho & =\sum_{n \in 2 \mathbf{Z}+m+i} \Psi_{\varepsilon}^{*}(u) T\left(u_{0}\right)_{m n} \frac{\rho_{m n}}{[m]^{\prime}} T\left(u_{0}\right)^{m n} \\
& =\sum_{n \in 2 \mathbf{Z}+m+i} \sum_{m^{\prime}=m \pm 1} \tilde{t}_{\varepsilon}^{*}\left(u-v_{0}\right)_{m^{\prime}}^{m} T\left(u_{0}\right)_{m^{\prime} n} \Psi^{*}(u)_{m}^{m^{\prime}} \frac{\rho_{m n}}{[m]^{\prime}} T\left(u_{0}\right)^{m n} \\
& =\sum_{n \in 2 \mathbf{Z}+m+i} \sum_{m^{\prime}=m \pm 1} \frac{\left[m^{\prime}\right]^{\prime}}{[m]^{\prime}} \tilde{t}_{\varepsilon}^{*}\left(u-v_{0}\right)_{m^{\prime}}^{m} T\left(u_{0}\right)_{m^{\prime} n} \frac{\rho_{m^{\prime} n}}{\left[m^{\prime}\right]^{\prime}} \Psi^{*}(u-2)_{m}^{m^{\prime}} T\left(u_{0}\right)^{m n} \\
& =\sum_{n \in 2 \mathbf{Z}+m+i} \sum_{m^{\prime}=m \pm 1} \sum_{\varepsilon^{\prime}} \frac{[m]^{\prime}}{[m]^{\prime}} \tilde{t}_{\varepsilon}^{*}\left(u-v_{0}\right)_{m^{\prime}}^{m} \tilde{t}_{\varepsilon^{\prime}}\left(u-v_{0}-2\right)_{m}^{m^{\prime}} T\left(u_{0}\right)_{m^{\prime} n} \frac{\rho_{m^{\prime} n}}{\left[m^{\prime}\right]^{\prime}} T\left(u_{0}\right)^{m n} \Psi_{\varepsilon^{\prime}}^{*}(u-2) \\
& =\sum_{m^{\prime}=m \pm 1} \sum_{n \in 2 \mathbf{Z}+m^{\prime}+1-i} \tilde{t}_{\varepsilon}^{*}\left(u-v_{0}\right)_{m^{\prime}}^{m} \tilde{t}_{\varepsilon^{\prime}}^{\prime}\left(u-v_{0}-2\right)_{m}^{m^{\prime}} T\left(u_{0}\right)_{m^{\prime} n} \frac{\rho_{m^{\prime} n}}{\left[m^{\prime}\right]^{\prime}} T\left(u_{0}\right)^{m n} \Psi_{\varepsilon^{\prime}}^{*}(u-2) \\
& =\sum_{m^{\prime}=m \pm 1} \sum_{\varepsilon^{\prime}} \tilde{t}_{\varepsilon}^{*}\left(u-v_{0}\right)_{m^{\prime}}^{m} \tilde{t}_{\varepsilon^{\prime}}^{\prime}\left(u-v_{0}-2\right)_{m}^{m^{\prime}} \rho \Psi_{\varepsilon^{\prime}}^{*}(u-2) \\
& =\rho \Psi_{\varepsilon^{\prime}}^{*}(u-2) .
\end{aligned}
$$

It only remains to check consistency with the normalization conditions. For example, let us multiplicate the second equation of $(3.7)$ by $T\left(u_{0}\right)^{m n^{\prime}}$ from the left:

$$
\begin{aligned}
T\left(u_{0}\right)^{m n} \Phi_{\varepsilon_{1}}(u) \Phi_{\varepsilon_{2}}^{*}(u) & =T\left(u_{0}\right)^{m n} \Phi_{\varepsilon_{1}}(u) \Phi_{-\varepsilon_{2}}(u-1) \\
& =\sum_{n^{\prime}, n^{\prime \prime}} t_{\varepsilon_{1}}\left(u-u_{0}\right)_{n^{\prime}}^{n} t_{-\varepsilon_{2}}\left(u-u_{0}-1\right)_{n^{\prime \prime}}^{n^{\prime}} \Phi(u)_{n^{\prime}}^{n} \Phi(u-1)_{n^{\prime \prime}}^{n^{\prime}} T\left(u_{0}\right)^{m n^{\prime \prime}} \\
& =\sum_{n^{\prime}, n^{\prime \prime}}(-1)^{n^{\prime}-m_{0}} \frac{n^{\prime}-n^{\prime \prime}}{\left[n^{\prime \prime}\right]} t_{\varepsilon_{1}}\left(u-u_{0}\right)_{n^{\prime}}^{n} t_{-\varepsilon_{2}}\left(u-u_{0}-1\right)_{n^{\prime \prime}}^{n^{\prime}} \Phi(u)_{n^{\prime}}^{n} \Phi^{*}(u)_{n^{\prime \prime}}^{n^{\prime}} T\left(u_{0}\right)^{m n^{\prime \prime}} \\
& =\sum_{n^{\prime}} t_{\varepsilon_{1}}\left(u-u_{0}\right)_{n^{\prime}}^{n} t_{\varepsilon_{2}}^{*}\left(u-u_{0}\right)_{n}^{n^{\prime}} \\
& =\delta_{\varepsilon_{1} \varepsilon_{2}} .
\end{aligned}
$$

The normalization condition for $\Psi_{\mathrm{s}}$ is checked similarly. 


\section{Appendix B. Normal forms and commutation relations}

Here some normal forms of operator products and some commutation relations are listed for the operators $V, \bar{V}, \tilde{V}, \tilde{\bar{V}}, W$. Let $V_{i}(u)$ be some vertex operators of the form (5.7). Then

$$
V_{i}\left(u^{\prime}\right) V_{j}(u)=z^{\prime \alpha_{i} \alpha_{j}}: V_{i}\left(u^{\prime}\right) V_{j}(u): f_{i j}\left(u-u^{\prime}\right)
$$

with

$$
\log f_{i j}(u)=\left\langle\phi_{i}^{-}(0) \phi_{j}^{+}(u)\right\rangle .
$$

For the operators with $A_{k}=-A_{-k}\left(\right.$ like $V(u), \bar{V}(u), \tilde{V}(u), \tilde{\bar{V}}(u)$, and $W(u)$ but not $W_{ \pm}(u)$ ) we have

$$
f_{i j}(u)=f_{j i}(u) .
$$

The commutation relations are given by

$$
V_{i}\left(u^{\prime}\right) V_{j}(u)=\left(\frac{z^{\prime}}{z}\right)^{\alpha_{i} \alpha_{j}} \frac{f_{i j}\left(u-u^{\prime}\right)}{f_{j i}\left(u^{\prime}-u\right)} V_{j}(u) V_{i}\left(u^{\prime}\right), \quad\left(z=x^{2 u}, z^{\prime}=x^{2 u^{\prime}}\right)
$$

We have

$$
\alpha_{V}=-\sqrt{\frac{r-1}{2 r}}, \quad \alpha_{\tilde{V}}=\sqrt{\frac{r}{2(r-1)}}, \quad \alpha_{W}=\sqrt{\frac{2}{r(r-1)}}
$$

and

$$
\begin{aligned}
& f_{V V}(u)=g(z ; \epsilon, r), \\
& f_{V \bar{V}}(u)=g^{-1}(x z) g^{-1}\left(x^{-1} z\right)=\frac{\left(x^{2 r-1} z ; x^{2 r}\right)_{\infty}}{\left(x z ; x^{2 r}\right)_{\infty}}, \\
& f_{\bar{V} \bar{V}}(u)=g\left(x^{2} z\right) g^{2}(z) g\left(x^{-2} z\right)=(1-z) \frac{\left(x^{2} z ; x^{2 r}\right)_{\infty}}{\left(x^{2 r-2} z ; x^{2 r}\right)_{\infty}}, \\
& f_{\tilde{V} \tilde{V}}(u)=\tilde{g}(z)=(1-z) g^{-1}(z ; \epsilon, r-1) \\
& f_{\tilde{V} \tilde{\bar{V}}}(u)=\tilde{g}^{-1}(x z) \tilde{g}^{-1}\left(x^{-1} z\right)=\frac{\left(x^{2 r-1} z ; x^{2 r-2}\right)_{\infty}}{\left(x^{-1} z ; x^{2 r-2}\right)_{\infty}}, \\
& f_{\tilde{V} \tilde{\bar{V}}}(u)=\tilde{g}\left(x^{2} z\right) \tilde{g}^{2}(z) \tilde{g}\left(x^{-2} z\right)=(1-z) \frac{\left(x^{-2} z ; x^{2 r-2}\right)_{\infty}}{\left(x^{2 r} z ; x^{2 r-2}\right)_{\infty}}, \\
& f_{V \tilde{V}}(u)=\frac{\left(x^{3} z ; x^{4}\right)_{\infty}}{\left(x z ; x^{4}\right)_{\infty}} \\
& f_{V \tilde{V}}(u)=f_{\bar{V} \tilde{V}}(u)=f_{V \tilde{V}}^{-1}\left(u+\frac{1}{2}\right) f_{V \tilde{V}}^{-1}\left(u-\frac{1}{2}\right)=1-z \\
& f_{\bar{V} \tilde{V}}(u)=\frac{1}{\left(1-x^{-2} z\right)\left(1-x^{2} z\right)}, \\
& f_{V W}(u)=f_{V \tilde{V}}\left(u+\frac{r-1}{2}\right) f_{V \bar{V}}\left(u+\frac{r}{2}\right)=\frac{\left(x^{r-1} z ; x^{2 r}\right)_{\infty}}{\left(x^{r+1} z ; x^{2 r}\right)_{\infty}}, \\
& f_{\tilde{V} W}(u)=f_{V W}^{-1}\left(u+\frac{1}{2}\right) f_{V W}^{-1}\left(u-\frac{1}{2}\right)=\frac{\left(x^{r+2} z ; x^{2 r}\right)_{\infty}}{\left(x^{r-2} z ; x^{2 r}\right)_{\infty}} \\
& f_{\tilde{V} W}(u)=f_{\tilde{V} \tilde{V}}\left(u+\frac{r-1}{2}\right) f_{\tilde{V} \bar{V}}\left(u+\frac{r}{2}\right)=\frac{\left(x^{r} z ; x^{2 r-2}\right)_{\infty}}{\left(x^{r-2} z ; x^{2 r-2}\right)_{\infty}} \\
& f_{\tilde{V} W}(u)=f_{\tilde{V} W}^{-1}\left(u+\frac{1}{2}\right) f_{\tilde{V} W}^{-1}\left(u-\frac{1}{2}\right)=\frac{\left(x^{r-3} z ; x^{2 r-2}\right)_{\infty}}{\left(x^{r+1} z ; x^{2 r-2}\right)_{\infty}}
\end{aligned}
$$

with $g(z)=g(z ; \epsilon, r)$ from $(2.3)$. 
Now list the most important commutation relations:

$$
\begin{aligned}
V\left(u_{1}\right) V\left(u_{2}\right) & =R_{0}\left(u_{1}-u_{2}\right) V\left(u_{2}\right) V\left(u_{1}\right), \\
V\left(u_{1}\right) \bar{V}\left(u_{2}\right) & =-\frac{\left[u_{1}-u_{2}+\frac{1}{2}\right]}{\left[u_{1}-u_{2}-\frac{1}{2}\right]} \bar{V}\left(u_{2}\right) V\left(u_{1}\right), \\
\bar{V}\left(u_{1}\right) \bar{V}\left(u_{2}\right) & =\frac{\left[u_{1}-u_{2}-1\right]}{\left[u_{1}-u_{2}+1\right]} \bar{V}\left(u_{2}\right) \bar{V}\left(u_{1}\right), \\
\tilde{V}\left(u_{1}\right) \tilde{V}\left(u_{2}\right) & =-R_{0}\left(u_{2}-u_{1}, \epsilon, r-1\right) \tilde{V}\left(u_{2}\right) \tilde{V}\left(u_{1}\right), \\
\tilde{V}\left(u_{1}\right) \tilde{\bar{V}}\left(u_{2}\right) & =-\frac{\left[u_{1}-u_{2}-\frac{1}{2}\right]^{\prime}}{\left[u_{1}-u_{2}+\frac{1}{2}\right]^{\prime}} \tilde{\bar{V}}\left(u_{2}\right) \tilde{V}\left(u_{1}\right), \\
V\left(u_{1}\right) \tilde{V}\left(u_{2}\right) & =-\mathrm{i} \tau\left(u_{1}-u_{2}\right) \tilde{V}\left(u_{2}\right) V\left(u_{1}\right), \\
V\left(u_{1}\right) \tilde{\bar{V}}\left(u_{2}\right) & =-\tilde{\bar{V}}\left(u_{2}\right) V\left(u_{1}\right), \\
\bar{V}\left(u_{1}\right) \tilde{V}\left(u_{2}\right) & =-\tilde{V}\left(u_{2}\right) \bar{V}\left(u_{1}\right), \\
\bar{V}\left(u_{1}\right) \tilde{\bar{V}}\left(u_{2}\right) & =\tilde{\bar{V}}\left(u_{2}\right) \bar{V}\left(u_{1}\right), \\
\tilde{\bar{V}}\left(u_{1}\right) \tilde{\bar{V}}\left(u_{2}\right) & =\frac{\left[u_{1}-u_{2}+1\right]^{\prime}}{\left[u_{1}-u_{2}-1\right]^{\prime}} \tilde{\bar{V}}\left(u_{2}\right) \tilde{\bar{V}}\left(u_{1}\right), \\
V\left(u_{1}\right) W\left(u_{2}\right) & =\frac{\left[u_{1}-u_{2}+\frac{r+1}{2}\right]}{\left[u_{1}-u_{2}+\frac{r-1}{2}\right]} W\left(u_{2}\right) V\left(u_{1}\right), \\
\bar{V}\left(u_{1}\right) W\left(u_{2}\right) & =\frac{\left[u_{1}-u_{2}+\frac{r-2}{2}\right]}{\left[u_{1}-u_{2}+\frac{r+2}{2}\right]} W\left(u_{2}\right) \bar{V}\left(u_{1}\right), \\
\tilde{V}\left(u_{1}\right) W\left(u_{2}\right) & =\frac{\left[u_{1}-u_{2}+\frac{r-2}{2}\right]^{\prime}}{\left[u_{1}-u_{2}+\frac{r}{2}\right]^{\prime}} W\left(u_{2}\right) \tilde{V}\left(u_{1}\right), \\
\tilde{V}\left(u_{1}\right) W\left(u_{2}\right) & =\frac{\left[u_{1}-u_{2}+\frac{r+1}{2}\right]^{\prime}}{\left[u_{1}-u_{2}+\frac{r-3}{2}\right]^{\prime}} W\left(u_{2}\right) \tilde{\bar{V}}\left(u_{1}\right) .
\end{aligned}
$$

\section{Appendix C. Proof of some identities for screening operators}

Let us calculate the commutator $\left[\tilde{Y}\left(u^{\prime}\right), X(u)\right]$. Let $\hat{p}=\sqrt{2 r(r-1)} \mathcal{P}$. Consider the product

$$
\begin{aligned}
\tilde{Y}\left(u^{\prime}\right) X(u)= & \frac{\epsilon^{2}}{\eta \eta^{\prime}} \int_{C} \frac{d v}{\mathrm{i} \pi} \int_{C^{\prime}} \frac{d v^{\prime}}{\mathrm{i} \pi} \tilde{\bar{V}}\left(v^{\prime}\right) \bar{V}(v) \frac{\left[v^{\prime}-u^{\prime}+\frac{1}{2}+\hat{p}\right]^{\prime}}{\left[v^{\prime}-u^{\prime}-\frac{1}{2}\right]^{\prime}} \frac{\left[v-u+\frac{1}{2}-\hat{p}\right]}{\left[v-u-\frac{1}{2}\right]} \\
= & \frac{\epsilon^{2}}{4 \eta \eta^{\prime}} \int_{C} \frac{d v}{\mathrm{i} \pi} \int_{C^{\prime}} \frac{d v^{\prime}}{\mathrm{i} \pi}: \tilde{\bar{V}}\left(v^{\prime}\right) \bar{V}(v): \frac{1}{\operatorname{sh} \epsilon\left(v^{\prime}-v-\frac{1}{2}\right) \operatorname{sh} \epsilon\left(v^{\prime}-v+\frac{1}{2}\right)} \\
& \times \frac{\left[v^{\prime}-u^{\prime}+\frac{1}{2}+\hat{p}\right]^{\prime}}{\left[v^{\prime}-u^{\prime}-\frac{1}{2}\right]^{\prime}} \frac{\left[v-u+\frac{1}{2}-\hat{p}\right]}{\left[v-u-\frac{1}{2}\right]} .
\end{aligned}
$$

Here the contour $C$ goes along the imaginary axis to the left of the point $u+\frac{1}{2}$ and the contour $C^{\prime}$ goes to the right of $u^{\prime}+\frac{1}{2}$ and to the left of $v \pm \frac{1}{2}$. The product $X(u) \tilde{Y}\left(u^{\prime}\right)$ differs from (C.1) in that the contour for the variable $v^{\prime}$ goes to the right of $v+\frac{1}{2}$. Hence, $\left[\tilde{Y}\left(u^{\prime}\right), X(u)\right]$ is the integral of the form (C.1) but with the contour for $v^{\prime}$ enclosing poles $v \pm \frac{1}{2}$. By taking the integration in $v^{\prime}$ by residues we obtain

$$
\begin{aligned}
{\left[\tilde{Y}\left(u^{\prime}\right), X(u)\right]=} & \frac{\epsilon}{2 \eta \eta^{\prime} \operatorname{sh} \epsilon} \int_{C} \frac{d v}{\mathrm{i} \pi}\left(: \tilde{\bar{V}}\left(v+\frac{1}{2}\right) \bar{V}(v): \frac{\left[v-u^{\prime}-1+\hat{p}\right]^{\prime}}{\left[v-u^{\prime}\right]^{\prime}} \frac{\left[v-u+\frac{1}{2}-\hat{p}\right]}{\left[v-u-\frac{1}{2}\right]}\right. \\
& \left.-: \tilde{\bar{V}}\left(v-\frac{1}{2}\right) \bar{V}(v): \frac{\left[v-u^{\prime}-2+\hat{p}\right]^{\prime}}{\left[v-u^{\prime}-1\right]^{\prime}} \frac{\left[v-u+\frac{1}{2}-\hat{p}\right]}{\left[v-u-\frac{1}{2}\right]}\right) \\
= & \frac{\epsilon}{2 \eta \eta^{\prime} \operatorname{sh} \epsilon} \int_{C} \frac{d v}{\mathrm{i} \pi}\left(W\left(v+\frac{r}{2}\right) F\left(v+\frac{r}{2}, u, u^{\prime} ; \hat{p}\right)-W\left(v-\frac{r}{2}\right) F\left(v-\frac{r}{2}, u, u^{\prime} ; \hat{p}\right)\right) .
\end{aligned}
$$


Here $W(u)$ is the operator defined by $(4.17)$ and

$$
F\left(v, u, u^{\prime} ; \hat{p}\right)=\frac{\left[v-u^{\prime}-\frac{r}{2}+1+\hat{p}\right]^{\prime}}{\left[v-u^{\prime}-\frac{r}{2}\right]^{\prime}} \frac{\left[v-u-\frac{r-1}{2}-\hat{p}\right]}{\left[v-u-\frac{r+1}{2}\right]} .
$$

The integrand in the last line of (C.2) is a total difference. It means that

$$
\left[\tilde{Y}\left(u^{\prime}\right), X(u)\right]=\frac{\epsilon}{\eta \eta^{\prime} \operatorname{sh} \epsilon} \oint \frac{d v}{2 \pi \mathrm{i}} W(v) F\left(v ; u, u^{\prime}, \hat{p}\right) .
$$

There are two poles inside the contour of integration: $u^{\prime}+\frac{r}{2}$ and $u-\frac{r-1}{2}$ and we finally obtain

$$
\left[\tilde{Y}\left(u^{\prime}\right), X(u)\right]=\frac{\epsilon}{\eta \eta^{\prime} \operatorname{sh} \epsilon}\left(W_{+}\left(u^{\prime}\right) \frac{[\hat{p}-1]^{\prime}}{\partial[0]^{\prime}} \frac{\left[u^{\prime}-u+\frac{1}{2}-\hat{p}\right]}{\left[u^{\prime}-u-\frac{1}{2}\right]}+W_{-}(u) \frac{[\hat{p}-1]}{\partial[0]} \frac{\left[u^{\prime}-u+\frac{3}{2}-\hat{p}\right]^{\prime}}{\left[u^{\prime}-u+\frac{1}{2}\right]^{\prime}}\right)
$$

with $W_{ \pm}(u)$ from (4.16b). Taking into account that $[u+\hat{p}]=(-)^{m-n}[u+n]$ and $[u+\hat{p}]^{\prime}=(-)^{m-n}[u+m]^{\prime}$ on $\mathcal{F}_{m n}$, we obtain (4.16a).

Consider the limit $u^{\prime} \rightarrow u-\frac{1}{2}$. According to (C.4) the commutator has the pole at this point. This pole comes from pinching the contour $C^{\prime}$ in (C.1) between $v-\frac{1}{2}$ and $u^{\prime}+\frac{1}{2}$ for $v$ being in the vicinity of the pole $u+\frac{1}{2}$. Similarly, the pole at $u^{\prime} \rightarrow u+\frac{1}{2}$ comes from pinching the contour $C^{\prime}$ between $v+\frac{1}{2}$ and $u^{\prime}+\frac{1}{2}$ for $v$ being in the vicinity of $u+\frac{1}{2}$. For the product $X(u) \tilde{Y}\left(u^{\prime}\right)$ the contour for $v$ lies to the left of all poles and the contour for $v^{\prime}$ lies to the right of all poles. Without other operators these contours can be moved far from all poles. It means that the product $X(u) \tilde{Y}\left(u^{\prime}\right)$ is always regular, while the poles of (C.4) completely belong to the product $\tilde{Y}\left(u^{\prime}\right) X(u)$. This proves $(4.20)$.

Let us prove (4.22). Consider the product

$$
\begin{aligned}
\tilde{Y}^{k}\left(u^{\prime}\right) X^{l}(u)= & (-)^{\frac{1}{2} k(k-1)+\frac{1}{2} l(l-1)}\left(\frac{\epsilon}{\eta}\right)^{k}\left(\frac{\epsilon}{\eta^{\prime}}\right)^{l} \int_{C^{\prime \times k}} \frac{d^{k} w}{(i \pi)^{k}} \int_{C^{\times l}} \frac{d^{l} v}{(i \pi)^{l}} \tilde{\bar{V}}\left(w_{1}\right) \ldots \tilde{\bar{V}}\left(w_{k}\right) \bar{V}\left(v_{l}\right) \ldots \bar{V}\left(v_{1}\right) \\
& \times \prod_{a=1}^{k} \frac{\left[w_{a}-u^{\prime}+\hat{p}+\frac{1}{2}-2(k+1-a)\right]^{\prime}}{\left[w_{a}-u^{\prime}-\frac{1}{2}\right]^{\prime}} \prod_{b=1}^{l} \frac{\left[v_{b}-u-\frac{3}{2}-\hat{p}+2 b\right]}{\left[v_{b}-u-\frac{1}{2}\right]} .
\end{aligned}
$$

Because of the commutation relations (B.20) and (B.27) the integrand can be symmetrized in all $w$ 's and in all $v$ 's by use of the identity [15]

$$
\frac{1}{k !} \sum_{\sigma \in S_{k}} \prod_{i=1}^{k}\left[v_{\sigma(i)}-2 i+2\right]=\frac{[k] !}{k ![1]^{k}} \prod_{i<j} \frac{\left[v_{i}-v_{j}\right]}{\left[v_{i}-v_{j}-1\right]} \prod_{i=1}^{k}\left[v_{i}-k+1\right], \quad[k] !=\prod_{i=1}^{k}[k] .
$$

We obtain

$$
\begin{aligned}
& \tilde{Y}^{k}\left(u^{\prime}\right) X^{l}(u)= \\
& =(-)^{\frac{1}{2} k(k-1)+\frac{1}{2} l(l-1)} \frac{[k]^{\prime} ![l] !}{k ! l ![1]^{\prime k}[1]^{l}}\left(\frac{\epsilon}{\eta}\right)^{k}\left(\frac{\epsilon}{\eta^{\prime}}\right)^{l} \int_{C^{\prime \times k}} \frac{d^{k} w}{(i \pi)^{k}} \int_{C^{\times l}} \frac{d^{l} v}{(i \pi)^{l}} \tilde{\bar{V}}\left(w_{1}\right) \ldots \tilde{\bar{V}}\left(w_{k}\right) \bar{V}\left(v_{l}\right) \ldots \bar{V}\left(v_{1}\right) \\
& \quad \times \prod_{a=1}^{k} \frac{\left[w_{a}-u^{\prime}+\hat{p}-k-\frac{1}{2}\right]^{\prime}}{\left[w_{a}-u^{\prime}-\frac{1}{2}\right]^{\prime}} \prod_{b=1}^{l} \frac{\left[v_{b}-u-\hat{p}+l-\frac{1}{2}\right]}{\left[v_{b}-u-\frac{1}{2}\right]} \prod_{a<a^{\prime}} \frac{\left[w_{a}-w_{a^{\prime}}\right]^{\prime}}{\left[w_{a}-w_{a^{\prime}}+1\right]^{\prime}} \prod_{b<b^{\prime}} \frac{\left[v_{b}-v_{b^{\prime}}\right]}{\left[v_{b}-v_{b^{\prime}}+1\right]} .
\end{aligned}
$$

Let $u^{\prime} \rightarrow u-\frac{1}{2}$. If any of $w_{a}$ approaches $u^{\prime}+\frac{1}{2}$, the contours $C$ become pinched between the poles $w_{a}+\frac{1}{2}$ and $u+\frac{1}{2}$. It means that $C^{\prime}$ can be deformed into a sum of two contours $C_{l}^{\prime}$ that goes to the left of $u^{\prime}+\frac{1}{2}$ 
and a small circle $C_{0}^{\prime}$ around $u^{\prime}+\frac{1}{2}$. Let us calculate the integral

$$
\begin{aligned}
\int_{C^{\prime k-1}} & \frac{d^{k-1} w}{(\mathrm{i} \pi)^{k-1}} \oint_{C_{0}^{\prime}} \frac{d w_{k}}{\mathrm{i} \pi} \tilde{\bar{V}}\left(w_{1}\right) \ldots \tilde{\bar{V}}\left(w_{k-1}\right) \tilde{\bar{V}}\left(w_{k}\right) \prod_{a=1}^{k} \frac{\left[w_{a}-u^{\prime}+\hat{p}-k-\frac{1}{2}\right]^{\prime}}{\left[w_{a}-u^{\prime}-\frac{1}{2}\right]^{\prime}} \prod_{a<a^{\prime}}^{k} \frac{\left[w_{a}-w_{a^{\prime}}\right]^{\prime}}{\left[w_{a}-w_{a^{\prime}}+1\right]^{\prime}} \\
= & \int_{C^{\prime k-1}} \frac{d^{k-1} w}{(\mathrm{i} \pi)^{k-1}} \oint_{C_{0}^{\prime}} \frac{d w_{k}}{\mathrm{i} \pi} \tilde{\bar{V}}\left(w_{1}\right) \ldots \tilde{\bar{V}}\left(w_{k-1}\right) \tilde{\bar{V}}\left(w_{k}\right) \\
& \times \prod_{a=1}^{k-1} \frac{\left[w_{a}-u^{\prime}+\hat{p}-k-\frac{1}{2}\right]^{\prime}}{\left[w_{a}-u^{\prime}-\frac{1}{2}\right]^{\prime}} \prod_{a<a^{\prime}}^{k-1} \frac{\left[w_{a}-w_{a^{\prime}}\right]^{\prime}}{\left[w_{a}-w_{a^{\prime}}+1\right]^{\prime}} \frac{\left[w_{k}-u^{\prime}+m-k-\frac{1}{2}\right]^{\prime}}{\left[w_{k}-u^{\prime}-\frac{1}{2}\right]^{\prime}} \prod_{a=1}^{k-1} \frac{\left[w_{k}-w_{a}\right]^{\prime}}{\left[w_{k}-w_{a}-1\right]^{\prime}} \\
= & 2 \int_{C_{l}^{\prime k-1}} \frac{d^{k-1} w}{(\mathrm{i} \pi)^{k-1}} \tilde{\bar{V}}\left(w_{1}\right) \ldots \tilde{\bar{V}}\left(w_{k-1}\right) \tilde{\bar{V}}\left(u^{\prime}+\frac{1}{2}\right) \frac{[\hat{p}-k]^{\prime}}{\partial[0]^{\prime}} \prod_{a=1}^{k-1} \frac{\left[w_{a}-u^{\prime}+\hat{p}-k-\frac{1}{2}\right]^{\prime}}{\left[w_{a}-u^{\prime}+\frac{1}{2}\right]^{\prime}} \prod_{a<a^{\prime}}^{k-1} \frac{\left[w_{a}-w_{a^{\prime}}\right]^{\prime}}{\left[w_{a}-w_{a^{\prime}}+1\right]^{\prime}} \\
= & (-)^{\frac{1}{2}(k-1)(k-2)} \tilde{X}^{k-1}\left(u^{\prime}\right) \tilde{\bar{V}}\left(u^{\prime}+\frac{1}{2}\right)\left(\frac{\eta^{\prime}}{\epsilon}\right)^{k-1} \frac{(k-1) ![1]^{k-1}[\hat{p}-k]^{\prime}}{[k-1]^{\prime} ! \partial[0]^{\prime}} .
\end{aligned}
$$

We substituted $C^{\prime}$ to $C_{l}^{\prime}$ in the fourth line because the integrand no more has a pole at $u^{\prime}+\frac{1}{2}$. It means that one may substitute

$$
\int_{C^{\prime k}} \rightarrow \int_{C_{l}^{\prime k}}+\sum_{a=1}^{k} \int_{C_{l}^{\prime a-1} \times C_{0}^{\prime} \times C_{l}^{\prime a-1}} \rightarrow \int_{C_{l}^{\prime k}}+k \int_{C_{l}^{\prime k-1}} \int_{C_{0}^{\prime}}
$$

in (C.7).

Similarly, $C=C_{r}-C_{0}$ with $C_{r}$ going to the right of all poles and $C_{0}$ being a small circle around $u+\frac{1}{2}$. We obtain

$$
\begin{aligned}
& \int_{C^{l-1}} \frac{d^{l-1} v}{(\mathrm{i} \pi)^{l-1}} \oint_{C_{0}} \frac{d v_{l}}{\mathrm{i} \pi} \bar{V}\left(v_{l}\right) \bar{V}\left(v_{l-1}\right) \ldots \bar{V}\left(v_{1}\right) \prod_{b=1}^{l} \frac{\left[v_{b}-u-\hat{p}+l-\frac{1}{2}\right]}{\left[v_{b}-u-\frac{1}{2}\right]} \prod_{b<b^{\prime}}^{l} \frac{\left[v_{b}-v_{b^{\prime}}\right]}{\left[v_{b}-v_{b^{\prime}}+1\right]} \\
& \quad=2 \int_{C_{r}^{l-1}} \frac{d^{l-1} v}{(\mathrm{i} \pi)^{l-1}} \bar{V}\left(u+\frac{1}{2}\right) \bar{V}\left(v_{l-1}\right) \ldots \bar{V}\left(v_{1}\right) \frac{[l-\hat{p}]}{\partial[0]} \prod_{b=1}^{l-1} \frac{\left[v_{b}-u-n+l-\frac{1}{2}\right]}{\left[v_{b}-u+\frac{1}{2}\right]} \prod_{b<b^{\prime}}^{l-1} \frac{\left[v_{b}-v_{b^{\prime}}\right]}{\left[v_{b}-v_{b^{\prime}}+1\right]} \\
& =(-)^{\frac{1}{2}(l-1)(l-2)} \bar{V}\left(u+\frac{1}{2}\right) Y^{l-1}(u)\left(\frac{\eta}{\epsilon}\right)^{l-1} \frac{(l-1) ![1]^{l-1}[l-\hat{p}]}{[l-1] ! \partial[0]} .
\end{aligned}
$$

The situation is the same as for the integral in w's.

By substitute (C.8) and (C.9) into (C.7) we obtain

$$
\begin{aligned}
\tilde{Y}^{k}\left(u^{\prime}\right) X^{l}(u)= & \left(\tilde{X}^{k}\left(u^{\prime}-1\right)+(-)^{k-1} \tilde{X}^{k-1}\left(u^{\prime}\right) \tilde{\bar{V}}\left(u^{\prime}+\frac{1}{2}\right) \frac{\epsilon}{\eta^{\prime}} \frac{[k]^{\prime}[\hat{p}-k]^{\prime}}{[1]^{\prime k} \partial[0]^{\prime}}\right) \\
& \times\left(Y^{l}(u+1)+(-)^{l-1} \bar{V}\left(u+\frac{1}{2}\right) Y^{l-1}(u) \frac{\epsilon}{\eta} \frac{[l][\hat{p}-l]}{[1]^{l} \partial[0]}\right) \\
= & \tilde{X}^{k}\left(u^{\prime}-1\right) Y^{l}(u+1)+(-)^{k-1} \tilde{X}^{k}\left(u^{\prime}-1\right) \bar{V}\left(u+\frac{1}{2}\right) Y^{l-1}(u) \frac{\epsilon}{\eta} \frac{[l][\hat{p}-l]}{[1]^{l} \partial[0]} \\
& +(-)^{l-1} \tilde{X}^{k-1}\left(u^{\prime}\right) \tilde{\bar{V}}\left(u^{\prime}+\frac{1}{2}\right) Y^{l}(u+1) \frac{\epsilon}{\eta^{\prime}} \frac{[k]^{\prime}[\hat{p}-k]^{\prime}}{[1]^{\prime k} \partial[0]^{\prime}} \\
& +(-)^{k+l} \tilde{X}^{k-1}\left(u^{\prime}\right) \tilde{\bar{V}}\left(u^{\prime}+\frac{1}{2}\right) \bar{V}\left(u+\frac{1}{2}\right) Y^{l-1}(u) \frac{\epsilon^{2}}{\eta \eta^{\prime}} \frac{[k]^{\prime}[\hat{p}-k]^{\prime}}{[1]^{\prime k} \partial[0]^{\prime}} \frac{[l][\hat{p}-l]}{[1]^{l} \partial[0]}
\end{aligned}
$$

The first three terms in the last expression are regular, because the contour of integrations of $\tilde{X}$ 's can be moved left and those of $Y$ 's right far away from poles. The last term contains the product

$$
\begin{aligned}
\tilde{\bar{V}}\left(u^{\prime}+\frac{1}{2}\right) \bar{V}\left(u+\frac{1}{2}\right) & =: \tilde{\bar{V}}\left(u^{\prime}+\frac{1}{2}\right) \bar{V}\left(u+\frac{1}{2}\right): \frac{1}{4 \operatorname{sh} \epsilon\left(u^{\prime}-u-\frac{1}{2}\right) \operatorname{sh} \epsilon\left(u^{\prime}-u+\frac{1}{2}\right)} \\
& =-\frac{1}{u^{\prime}-u+\frac{1}{2}} \frac{W_{-}(u)}{4 \epsilon \operatorname{sh} \epsilon}+O(1) \quad \text { for } u^{\prime} \rightarrow u-\frac{1}{2} .
\end{aligned}
$$

Substituting (C.11) into (C.10) and restricting the result to $\mathcal{F}_{m n}$ we obtain (4.22). 


\section{Appendix D. Guidelines of the proof of (4.23)}

Eq. (4.23) can be checked as follows. Let us write out the equations (3.21b) for $\Delta u_{0}=-1 / 2$ explicitly:

$$
\begin{aligned}
\Psi^{*}(u)_{m-2 k}^{m-2 k+1} \Lambda\left(u_{0}\right)_{m}^{m-2 k}= & \Lambda\left(u_{0}\right)_{m-1}^{m-2 k+1} \Psi^{*}(u)_{m}^{m-1} \frac{\left[u_{0}-u-m+k-\frac{1}{2}\right]^{\prime}[k-1]^{\prime}}{\left[u_{0}-u-\frac{1}{2}\right]^{\prime}[m-1]^{\prime}} \\
& +\Lambda\left(u_{0}\right)_{m+1}^{m-2 k+1} \Psi^{*}(u)_{m}^{m+1} \frac{\left[u_{0}-u+k-\frac{1}{2}\right]^{\prime}[m-k+1]^{\prime}}{\left[u_{0}-u-\frac{1}{2}\right]^{\prime}[m+1]^{\prime}} \\
\Psi^{*}(u)_{m-2 k}^{m-2 k-1} \Lambda\left(u_{0}\right)_{m}^{m-2 k}= & \Lambda\left(u_{0}\right)_{m-1}^{m-2 k-1} \Psi^{*}(u)_{m}^{m-1} \frac{\left[u_{0}-u-k-\frac{1}{2}\right]^{\prime}[m-k-1]^{\prime}}{\left[u_{0}-u-\frac{1}{2}\right]^{\prime}[m-1]^{\prime}} \\
& +\Lambda\left(u_{0}\right)_{m+1}^{m-2 k-1} \Psi^{*}(u)_{m}^{m+1} \frac{\left[u_{0}-u+m-k-\frac{1}{2}\right]^{\prime}[k+1]^{\prime}}{\left[u_{0}-u-\frac{1}{2}\right]^{\prime}[m+1]^{\prime}}
\end{aligned}
$$

Let us substitute (4.11) and (4.23) into (D.1), then pull the operator $Y^{l-1}\left(u_{0}\right)$ to the right in each term and omit as a common factor. We obtain

$$
\begin{aligned}
\tilde{V}(u) \tilde{X}^{k-1}\left(u_{0}-\frac{1}{2}\right) W_{-}\left(u_{0}\right)= & \tilde{X}^{k-2}\left(u_{0}-\frac{1}{2}\right) W_{-}\left(u_{0}\right) \tilde{Y}(u) \tilde{V}(u) \frac{\left[u_{0}-u-m+k-\frac{1}{2}\right]^{\prime}[k+1]^{\prime}}{\left[u_{0}-u-\frac{1}{2}\right]^{\prime}[m]^{\prime}} \\
& +\tilde{X}^{k-1}\left(u_{0}-\frac{1}{2}\right) W_{-}\left(u_{0}\right) \tilde{V}(u) \frac{\left[u_{0}-u+k-\frac{1}{2}\right]^{\prime}[m-k+1]^{\prime}}{\left[u_{0}-u-\frac{1}{2}\right]^{\prime}[m]^{\prime}}, \\
\tilde{Y}(u) \tilde{V}(u) \tilde{X}^{k-1}\left(u_{0}-\frac{1}{2}\right) W_{-}\left(u_{0}\right)= & \tilde{X}^{k-1}\left(u_{0}-\frac{1}{2}\right) W_{-}\left(u_{0}\right) \tilde{Y}(u) \tilde{V}(u) \frac{\left[u_{0}-u-k-\frac{1}{2}\right]^{\prime}[m-k-1]^{\prime}}{\left[u_{0}-u-\frac{1}{2}\right]^{\prime}[m]^{\prime}} \\
& +\tilde{X}^{k} W_{-}\left(u_{0}\right) \tilde{V}(u) \frac{\left[u_{0}-u+m-k-\frac{1}{2}\right]^{\prime}[k+1]^{\prime}}{\left[u_{0}-u-\frac{1}{2}\right]^{\prime}[m]^{\prime}} .
\end{aligned}
$$

It can be checked that all integration contours in $\tilde{X}$ 's and $\tilde{Y}$ 's in all terms can be deformed to the same curve. It means that we shall prove (D.1) if we check equality of the integrands symmetrized in all integration variables.

To do it, let us substitute definitions of the screening operators into these equations. Then we have to render the products of the operators to any given order by use of commutation relations (B.22), (B.27), (B.29), and (B.31). For example, the first term in the r. h. s. of the first equation is proportional to

$$
\begin{gathered}
\frac{\left[u_{0}-u-m+k-\frac{1}{2}\right]^{\prime}[k-1]^{\prime}}{\left[u_{0}-u-\frac{1}{2}\right]^{]}[m]^{\prime}} \int \frac{d^{k-1} w}{(\mathrm{i} \pi)^{k-1}} \tilde{\bar{V}}\left(w_{k-1}\right) \ldots \tilde{\bar{V}}\left(w_{2}\right) \tilde{\bar{V}}\left(w_{1}\right) W_{-}\left(u_{0}\right) \tilde{V}(u) \\
\times \frac{\left[w_{1}-u_{0}+1\right]^{\prime}}{\left[w_{1}-u_{0}-1\right]^{\prime}} \frac{\left[w_{1}-u-\frac{1}{2}+m\right]^{\prime}}{\left[w_{1}-u-\frac{1}{2}\right]^{\prime}} \prod_{a=2}^{k-1} \frac{\left[w_{a}-u_{0}+m-1-2(a-2)\right]^{\prime}}{\left[w_{a}-u_{0}+1\right]^{\prime}} .
\end{gathered}
$$

The next step is to symmetrize the integrand. For symmetrization in $w_{2}, \ldots, w_{k-1}$ the identity (C.6) can be applied. Symmetrization in the position of the remaining variable $w_{1}$ produces the sum:

$$
\begin{aligned}
(D .2)= & \frac{\left[u_{0}-u-m+k-\frac{1}{2}\right]^{\prime}[1]^{\prime}}{\left[u_{0}-u-\frac{1}{2}\right]^{\prime}[m]^{\prime}} \frac{[k-1]^{\prime} !}{(k-1) ![1]^{k-1}} \sum_{j=1}^{k-1}\left(\frac{\left[w_{j}-u_{0}-1\right]^{\prime}}{\left[w_{j}-u_{0}+1\right]^{\prime}} \frac{\left[w_{j}-u-\frac{1}{2}+m\right]^{\prime}}{\left[w_{j}-u-\frac{1}{2}\right]^{\prime}}\right. \\
& \left.\times \prod_{\substack{a<a^{\prime} \\
a, a^{\prime} \neq j}}^{k-1} \frac{\left[w_{a}-w_{a^{\prime}}\right]^{\prime}}{\left[w_{a}-w_{a^{\prime}}-1\right]^{\prime}} \prod_{\substack{a=1 \\
a \neq j}}^{k-1} \frac{\left[w_{a}-u_{0}+m-k\right]^{\prime}}{\left[w_{a}-u_{0}+1\right]^{\prime}} \prod_{a=1}^{j-1} \frac{\left[w_{j}-w_{a}-1\right]^{\prime}}{\left[w_{j}-w_{a}+1\right]^{\prime}}\right) .
\end{aligned}
$$

The same should be done for every other term. By equating the integrands in both sides, we obtain some equalities. To shorten the formulas let us introduce notations $z_{a}=w_{a}-u-\frac{1}{2}, z_{0}=u_{0}-u-\frac{1}{2}$ and assign 
$z_{a b}=z_{a}-z_{b}, z_{a 0}=z_{a}-z_{0}$. We have

$$
\begin{aligned}
& \prod_{a<a^{\prime}}^{k-1} \frac{\left[z_{a a^{\prime}}\right]^{\prime}}{\left[z_{a a^{\prime}}-1\right]^{\prime}} \prod_{a=1}^{k-1} \frac{\left[z_{a 0}+m-k\right]^{\prime}}{\left[z_{a 0}+1\right]^{\prime}} \frac{\left[z_{a}+1\right]^{\prime}}{\left[z_{a}\right]^{\prime}} \\
& =\frac{\left[z_{0}-m+k\right]^{\prime}[1]^{\prime}}{\left[z_{0}+1\right]^{\prime}[m]^{\prime}} \sum_{j=1}^{k-1} \prod_{\substack{a<a^{\prime} \\
a, a^{\prime} \neq j}}^{k-1} \frac{\left[z_{a a^{\prime}}\right]^{\prime}}{\left[z_{a a^{\prime}}-1\right]^{\prime}} \prod_{\substack{a=1 \\
a \neq j}}^{k-1} \frac{\left[z_{a 0}+m-k\right]^{\prime}}{\left[z_{a 0}+1\right]^{\prime}} \prod_{a=1}^{j-1} \frac{\left[z_{j a}-1\right]^{\prime}}{\left[z_{j a}+1\right]^{\prime}} \\
& +\frac{\left[z_{0}+k\right]^{\prime}[m-k+1]^{\prime}}{\left[z_{0}+1\right]^{\prime}[m]^{\prime}} \prod_{a<a^{\prime}}^{k-1} \frac{\left[z_{a a^{\prime}}\right]^{\prime}}{\left[z_{a a^{\prime}}+1\right]^{\prime}} \prod_{a=1}^{k-1} \frac{\left[z_{a 0}+m-k+1\right]^{\prime}}{\left[z_{a 0}+1\right]^{\prime}}, \\
& \frac{[m]^{\prime}}{[k]^{\prime}} \sum_{j=1}^{k} \frac{\left[z_{j}-2 k+m\right]^{\prime}}{\left[z_{j}\right]^{\prime}} \prod_{\substack{a<a^{\prime} \\
a, a^{\prime} \neq j}}^{k} \frac{\left[z_{a a^{\prime}}\right]^{\prime}}{\left[z_{a a^{\prime}}-1\right]^{\prime}} \prod_{\substack{a=1 \\
a \neq j}}^{k} \frac{\left[z_{a 0}+m-k\right]^{\prime}}{\left[z_{a 0}+1\right]^{\prime}} \frac{\left[z_{a}+1\right]^{\prime}}{\left[z_{a}\right]^{\prime}} \prod_{a=j+1}^{k} \frac{\left[z_{a j}-1\right]^{\prime}}{\left[z_{a j}+1\right]^{\prime}} \\
& =\frac{\left[z_{0}-k\right]^{\prime}[m-k-1]^{\prime}}{[k]^{\prime}\left[z_{0}+1\right]^{\prime}} \sum_{j=1}^{k} \frac{\left[z_{j}+m\right]^{\prime}}{\left[z_{j}\right]^{\prime}} \prod_{\substack{a<a^{\prime} \\
a, a^{\prime} \neq j}}^{k} \frac{\left[z_{a a^{\prime}}\right]^{\prime}}{\left[z_{a a^{\prime}}-1\right]^{\prime}} \prod_{\substack{a=1 \\
a \neq j}}^{k} \frac{\left[z_{a 0}+m-k-1\right]^{\prime}}{\left[z_{a 0}+1\right]^{\prime}} \prod_{a=1}^{j-1} \frac{\left[z_{a j}-1\right]^{\prime}}{\left[z_{a j}+1\right]^{\prime}} \\
& +\frac{\left[z_{0}+m-k\right]^{\prime}[k+1]^{\prime}}{[1]^{\prime}\left[z_{0}+1\right]^{\prime}} \prod_{a=1}^{k} \frac{\left[z_{a 0}+m-k\right]^{\prime}}{\left[z_{a 0}+1\right]^{\prime}} \prod_{a<a^{\prime}}^{k} \frac{\left[z_{a a^{\prime}}\right]^{\prime}}{\left[z_{a a^{\prime}}+1\right]^{\prime}} .
\end{aligned}
$$

These identities are proven by comparing residues of poles by induction along the guidelines of Appendix C of [1]. The proof is straightforward but very cumbersome and we omit it.

\section{Appendix E. Trace functions}

Here we list the constants $c_{i}$ and functions $g_{i j}(u)$ defined by (5.10) for the operators $V(u), \tilde{V}(u)$ and $W_{-}$. We assume that $z=x^{2 u}$.

$$
\begin{aligned}
c_{V}= & \frac{\left(x^{6} ; x^{4}, x^{4}, x^{2 r}\right)_{\infty}\left(x^{2 r+6} ; x^{4}, x^{4}, x^{2 r}\right)_{\infty}}{\left(x^{8} ; x^{4}, x^{4}, x^{2 r}\right)_{\infty}\left(x^{2 r+4} ; x^{4}, x^{4}, x^{2 r}\right)_{\infty}}, \\
c_{\tilde{V}}= & \frac{\left(x^{4} ; x^{4}, x^{4}, x^{2 r-2}\right)_{\infty}\left(x^{2 r+6} ; x^{4}, x^{4}, x^{2 r-2}\right)_{\infty}}{\left(x^{6} ; x^{4}, x^{4}, x^{2 r-2}\right)_{\infty}\left(x^{2 r+4} ; x^{4}, x^{4}, x^{2 r-2}\right)_{\infty}}, \\
c_{W}= & \frac{\left(x^{2} ; x^{2 r-2}\right)_{\infty}}{\left(x^{2} ; x^{2 r}\right)_{\infty}}, \\
g_{V V}(u)= & \frac{\left(x^{2} z ; x^{4}, x^{4}, x^{2 r}\right)_{\infty}\left(x^{2 r+2} z ; x^{4}, x^{4}, x^{2 r}\right)_{\infty}\left(x^{6} z^{-1} ; x^{4}, x^{4}, x^{2 r}\right)_{\infty}\left(x^{2 r+6} z^{-1} ; x^{4}, x^{4}, x^{2 r}\right)_{\infty}}{\left(x^{4} z ; x^{4}, x^{4}, x^{2 r}\right)_{\infty}\left(x^{2 r} z ; x^{4}, x^{4}, x^{2 r}\right)_{\infty}\left(x^{8} z^{-1} ; x^{4}, x^{4}, x^{2 r}\right)_{\infty}\left(x^{2 r+4} z^{-1} ; x^{4}, x^{4}, x^{2 r}\right)_{\infty}}(\mathrm{E} .2) \\
g_{\tilde{V} \tilde{V}}(u)= & \frac{\left(z ; x^{4}, x^{4}, x^{2 r-2}\right)_{\infty}\left(x^{2 r+2} z ; x^{4}, x^{4}, x^{2 r-2}\right)_{\infty}\left(x^{4} z^{-1} ; x^{4}, x^{4}, x^{2 r-2}\right)_{\infty}\left(x^{2 r+6} z^{-1} ; x^{4}, x^{4}, x^{2 r-2}\right)_{\infty}}{\left(x^{2} z ; x^{4}, x^{4}, x^{2 r-2}\right)_{\infty}\left(x^{2 r} z ; x^{4}, x^{4}, x^{2 r-2}\right)_{\infty}\left(x^{6} z^{-1} ; x^{4}, x^{4}, x^{2 r-2}\right)_{\infty}\left(x^{2 r+4} z^{-1} ; x^{4}, x^{4}, x^{2 r-2}\right)_{\infty}}, \\
g_{\tilde{V} V}(u)= & \frac{\left(x^{3} z ; x^{4}, x^{4}\right)_{\infty}\left(x^{7} z^{-1} ; x^{4}, x^{4}\right)_{\infty}}{\left(x z ; x^{4}, x^{4}\right)_{\infty}\left(x^{5} z^{-1} ; x^{4}, x^{4}\right)_{\infty}}, \\
g_{V W_{-}}(u)= & \frac{\left(z ; x^{4}, x^{2 r}\right)_{\infty}\left(x^{2 r+2} z^{-1} ; x^{4}, x^{2 r}\right)_{\infty}}{\left(x^{2} z ; x^{4}, x^{2 r}\right)_{\infty}\left(x^{2 r+4} z^{-1} ; x^{4}, x^{2 r}\right)_{\infty}} \\
g_{\tilde{V} W_{-}}(u)= & \frac{\left(x z ; x^{4}, x^{2 r-2}\right)_{\infty}\left(x^{2 r+3} z^{-1} ; x^{4}, x^{2 r-2}\right)_{\infty}}{\left(x^{-1} z ; x^{4}, x^{2 r-2}\right)_{\infty}\left(x^{2 r+1} z^{-1} ; x^{4}, x^{2 r-2}\right)_{\infty}} .
\end{aligned}
$$

List also some important combinations

$$
g_{V V}(1)=\frac{\left(x^{4} ; x^{4}, x^{4}, x^{2 r}\right)_{\infty}^{2}\left(x^{2 r+4} ; x^{4}, x^{4}, x^{2 r}\right)_{\infty}^{2}}{\left(x^{6} ; x^{4}, x^{4}, x^{2 r}\right)_{\infty}^{2}\left(x^{2 r+2} ; x^{4}, x^{4}, x^{2 r}\right)_{\infty}^{2}}
$$




$$
\begin{aligned}
g_{\tilde{V} V}(u) g_{\tilde{V} V}(u-1) & =\frac{1}{\left(x^{-1} z ; x^{4}\right)_{\infty}\left(x^{5} z^{-1} ; x^{4}\right)_{\infty}}=x^{\frac{1}{2}\left(u-\frac{1}{2}\right)\left(u-\frac{5}{2}\right)} \frac{\left(x^{4}, x^{4}\right)_{\infty}}{\theta_{1}\left(\frac{u}{2}-\frac{1}{4} ; \frac{i \pi}{2 \epsilon}\right)}, \\
g_{V W_{-}}(u) g_{V W_{-}}(u+1) & =x^{-u^{2} / r+u} \frac{[u]}{\left(x^{2 r} ; x^{2 r}\right)_{\infty}} .
\end{aligned}
$$

\section{References}

1. M. Lashkevich and Ya. Pugai, Nucl. Phys. B516 [FS] (1998) 623 [hep-th/9710099]

2. M. Lashkevich and Ya. Pugai, JETP Lett. 68 (1998) 257 [hep-th/9805216]

3. M. Jimbo and T. Miwa, Algebraic analysis of solvable lattice models, CBMS Regional Conference Series in Mathematics 85 AMS (1994)

4. R. J. Baxter, Ann. Phys. 76 (1973) 1; 26; 48

5. O. Foda, K. Iohara, M. Jimbo, R. Kedem, T. Miwa, and H. Yan, Prog. Theor. Phys. Suppl. 118 (1995) 1 [hep-th/9405058]

6. S. Lukyanov and Ya. Pugai, Nucl. Phys. B473 [FS] (1996) 631 [hep-th/9602074]

7. B. Sutherland, J. Math. Phys. 11 (1970) 3183; C. Fan and F. Y. Wu, Phys. Rev. B2 (1970) 723

8. R. J. Baxter, Phil. Trans. Royal Soc. London 289 (1978) 315

9. R. J. Baxter, Phys. Rev. Lett. 26 (1971) 832; Ann. Phys. (N.Y.) 70 (1972) 193

10. M. Jimbo, T. Miwa, and A. Nakayashiki, J. Phys. A26 (1993) 2199

11. M. Jimbo, T. Miwa, and Y. Ohta, Int. J. Mod. Phys. A8 (1993) 1457

12. B. Davies, O. Foda, M. Jimbo, T. Miwa, and A. Nakayashiki, J. Phys. A26 (1993) 2199

13. F. A. Smirnov, Form factors in completely integrable models of quantum field theory, World Scientific, Singapore (1992)

14. R. J. Baxter and S. B. Kelland, J. Phys. C7 (1974) L403

15. M. Jimbo, M. Lashkevich, T. Miwa, and Ya. Pugai, Phys. Lett. A229 (1997) 285 [hep-th/9607177]

16. Y. Hara, Nucl. Phys. B572 [FS] (2000) 574 [math-ph/9910046]

17. S.-J. Kang, M. Kashiwara, K. Misra, T. Miwa, T. Nakashima, and A. Nakayashiki, Int. J. Mod. Phys. A7 (Suppl. 1A) (1992) 449

18. C. Frønsdal, Publ. RIMS, Kyoto Univ. 33 (1997) 91 [q-alg/9606020]; Lett. Math. Phys. 40 (1997) 117 [q-alg/9611028]

19. M. Jimbo, H. Konno, S. Odake, and J. Shiraishi, preprint q-alg/9712029

20. N. Kitanine, J. M. Maillet, and V. Terras, Nucl. Phys. B554 (1999) 647 [math-ph/9807020]; preprint math-ph/9907019

21. A. G. Izergin, N. Kitanine, J. M. Maillet, and V. Terras, preprint solv-int/9812021

22. A. Nakayashiki, S. Pakuliak, and V. Tarasov, preprint q-alg/9712002 